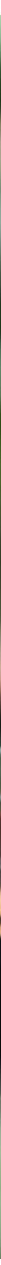

\title{
Procès-verbal de la deuxième Chambre médicale de 2015
}

\section{Monika Henzen}

Cheffe du Secrétariat central

Mercredi 28 octobre 2015

\section{Accueil, communications, désignation du bureau}

Jürg Schlup/président de la FMH souhaite la bienvenue aux délégués à la deuxième Chambre médicale de cette année. Suite au mandat donné par la ChM du 7 mai 2015 au Comité central, la séance d'automne a été prolongée d'une demi-journée; les mesures de stabilisation budgétaire et les autres objets tels que les élections, les modifications statutaires, la révision tarifaire, l'intégration de l'IPI ne pouvant être traités en une seule journée.
Le président souhaite également la bienvenue aux invités Jean Pierre Keller/vice-président de l'ISFM et Raphael Stolz/vice-président de l'ISFM.

Anne-Geneviève Bütikofer/secrétaire générale de la FMH donne les informations d'usage pour le déroulement de la séance et présente les différents documents qui ont été distribués aux délégués à leur arrivée.

Le président désigne ensuite le Bureau de la Chambre médicale, qui se compose du président, de Christine Romann/CC FMH, de la secrétaire générale, de Christoph Kreyden/responsable des élections, épaulé par la juriste Barbara Linder/ISFM, et des personnes suivantes qu'il propose d'élire comme scrutateurs: 


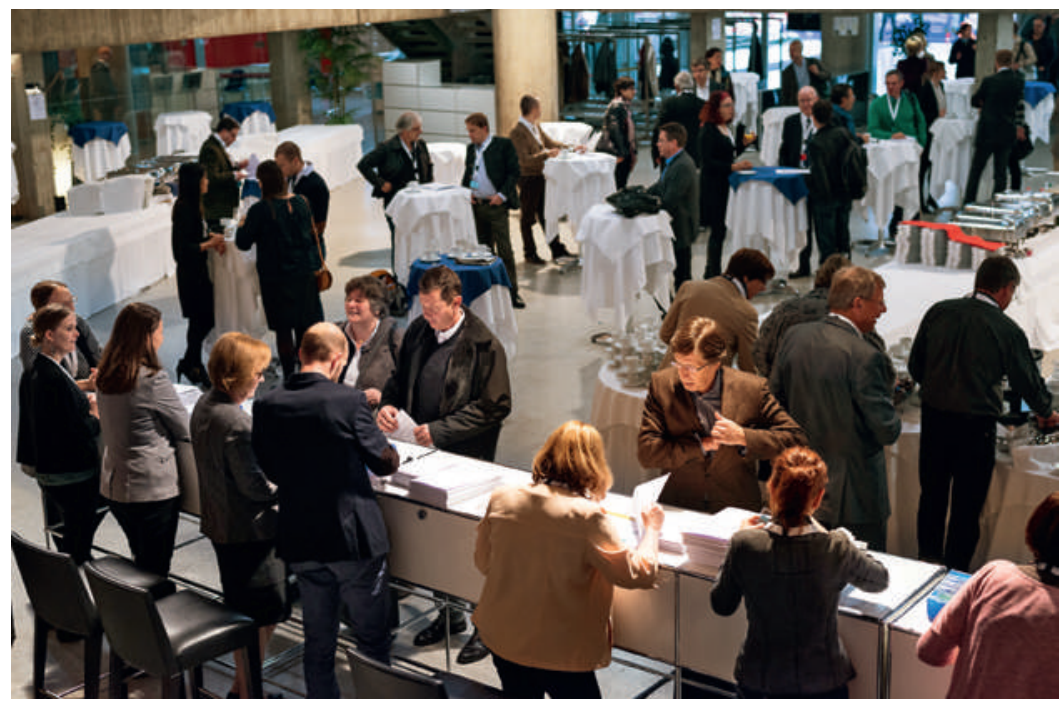

Travail diligent à l'accueil pour remettre leur carte de vote et les documents de séance aux délégués venus de toute la Suisse.

Jean-Claude Brückner, Lukas Meier, Karl-Olof Lövblad, Josef Brandenberg, Josef Widler, Daniel Schröpfer et Véronique Monnier-Cornuz.

Les scrutateurs sont élus par 100 voix, 2 non et 1 abstention.

\section{Ordre du jour}

L'ordre du jour est accepté à l'unanimité.

Le président propose de clore la séance d'aujourd'hui à $18 \mathrm{~h} 30$ au plus tard.

\section{Décision:}

La proposition de clore la séance à $18 \mathrm{~h} 30$ est acceptée par 100 oui et 3 abstentions.

Conformément à l'art. 11 al. 3 RE, aucune décision ne peut être prise et aucune élection ne peut avoir lieu après $18 \mathrm{~h} 30$. Si le quorum n'est plus atteint avant $18 \mathrm{~h} 30$, les points en suspens seraient repoussés au lendemain, jeudi 29 octobre 2015.

Le président dépose deux motions d'ordre.

Conformément à l'art. 11 al. 7 RE, il est possible de limiter le temps de parole sur proposition de la Chambre médicale ou du président.

Les délégués, les membres du Comité central et la secrétaire générale ont chacun le droit, conformément à l'art. 11 al. $5 \mathrm{RE}$, de faire des propositions quant aux objets à l'ordre du jour soumis à discussion. Le texte des propositions et amendements doit être présenté au président par écrit ou exceptionnellement par oral, avant ou pendant la séance.

Motion d'ordre 1: temps de parole limité

Pour la durée du point 2 «Mesures de stabilisation budgétaire», le temps de parole est limité à deux minutes par orateur. Aucune limite n'est prononcée pour l'orateur désigné par le président de cha- cune des organisations médicales ayant droit de vote ou pour l'intervenant du Comité central.

Décision:

La motion d'ordre est acceptée par 100 oui, 3 non et

2 abstentions.

Motion d'ordre 2: propositions soumises par écrit Pour le point 2 «Mesures de stabilisation budgétaire", les propositions et amendements doivent être présentés par écrit au président. Les propositions orales ne sont pas acceptées et ne sont pas valides.

Décision:

La proposition est acceptée par 100 oui, 5 non et 8 abstentions.

\section{Mesures de stabilisation budgétaire}

En introduction, Jürg Schlup/président de la FMH souligne qu'un équilibre financier est nécessaire entre les dépenses et projets en cours et les recettes pour que la FMH reste un partenaire central et crédible du domaine de la santé. Seules des finances équilibrées permettent de garantir à la FMH de continuer de se concentrer sur ses activités clés et de les mettre en œuvre. La FMH ne possède pas d'argent propre, elle ne fait que gérer l'argent de ses membres. Il rappelle le mandat exprimé par la ChM, le 30 octobre 2014, selon lequel les délégués avaient décidé la création d'une taskforce qui devait proposer des mesures visant à équilibrer durablement les dépenses et les recettes de la FMH et à créer une marge de manœuvre financière pour la mise en œuvre de projets prioritaires. Dans ce contexte, il s'est agi de passer en revue les tâches et activités de la FMH et d'en fixer les priorités. A la Chambre médicale du 7 mai 2015, les délégués ont pris connaissance du rapport et des mesures proposées par la taskforce et ils ont finalement demandé au Comité central et à la Secrétaire générale de présenter à la prochaine $\mathrm{ChM}$ des mesures réalistes et réalisables.

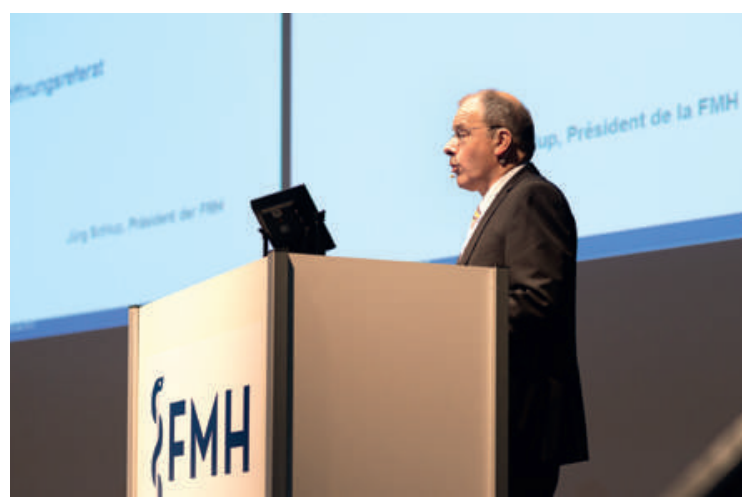

Le président de la FMH Jürg Schlup ouvre la deuxième Chambre médicale de l'année par un exposé. 
Le Comité central entend assumer ses responsabilités et faire face aux défis à relever. Le rapport de KPMG a été analysé avec toutes les divisions internes. Alors que le rapport de KPMG a décelé un potentiel d'amélioration de CHF 5,4 millions, le Comité central voit après analyse un potentiel de CHF 6,2 millions, par une réduction des coûts de CHF 4,3 millions et par des recettes supplémentaires de CHF 1,9 million. Les mesures approuvées seront mises en œuvre en deux étapes: une amélioration du résultat de CHF 3,3 millions doit avoir lieu en 2016, et de CHF 2,9 millions entre 2017 et 2019.

L'Assemblée des délégués et la Commission de gestion ont été informées au préalable du rapport et du catalogue de mesures soumis aujourd'hui au vote. Le Comité central considère que les mesures proposées sont appropriées et défendables. Le président, en son nom et en celui du Comité central, invite vivement les délégués à prendre les mesures qui s'imposent dès aujourd'hui et non dans six mois. En remettant leur décision à une date ultérieure, ils bloqueraient les travaux du Comité central et la suppression d'une seule des 11 mesures principales mettrait en péril l'objectif d'un budget équilibré.

Anne-Geneviève Bütikofer/secrétaire générale de la FMH précise la démarche et la méthode de travail. Le Comité central a privilégié une approche basée sur la pertinence et la priorité des activités (mission/vision) plutôt que de se concentrer sur la seule réduction des dépenses et l'augmentation des recettes. Les propositions faites

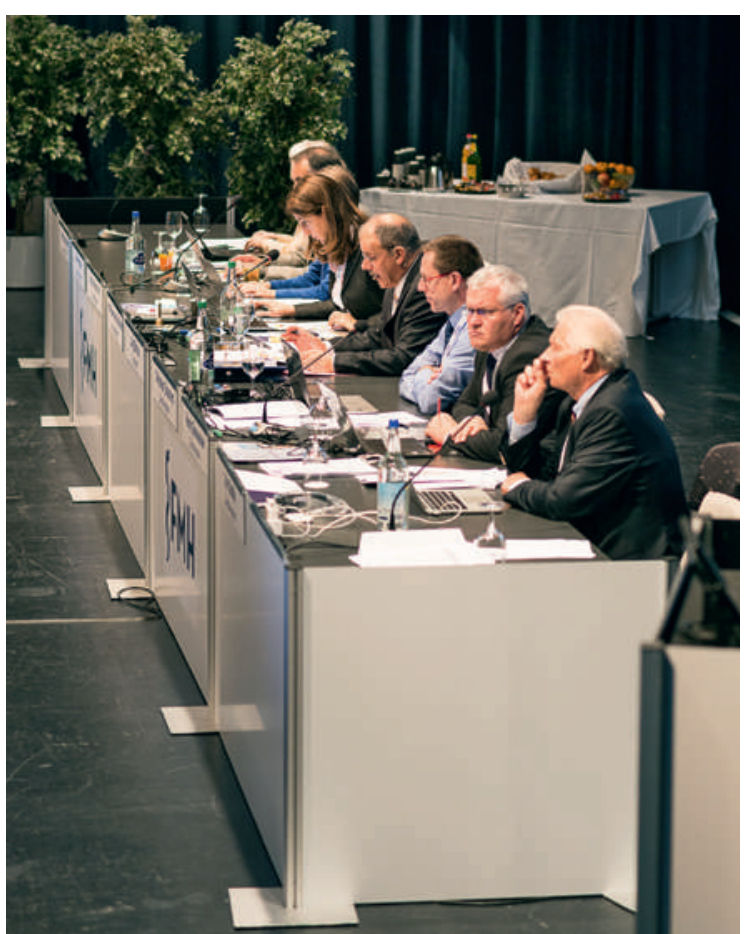

Le Comité central in corpore suit avec attention les débats depuis le podium. par KPMG ont été vérifiées, analysées et les mesures ont été soit reprises, soit corrigées ou encore redéveloppées. Les divisions et services ont été associés à ce processus permettant ainsi d'ajouter des mesures supplémentaires au rapport de KPMG. Tout ce travail a débouché sur environ 120 mesures offrant un abaissement des charges ou de nouvelles sources de recettes. A elles seules, les 11 mesures principales présentent un potentiel d'économies de CHF 2,767 millions. Contrairement au rapport de KPMG, le Comité central a identifié des possibilités de recettes supplémentaires correspondant à $13 \%$ du potentiel d'économies. Une hausse des cotisations de membres est cependant nécessaire car les coûts pour les prestations fournies sont aujourd'hui plus élevés. Par ailleurs, le Comité central a l'intention de créer un pooling de projets, visant à garantir la réalisation ciblée de projets stratégiques, dont l'utilisation sera définie par un règlement qui est en cours d'élaboration. Il est important de signaler que les mesures d'économie n'entraînent pas de suppressions d'emplois directes. Les ressources libérées donneront la possibilité aux employés de la FMH de s'engager sur de nouveaux projets et de réduire les heures supplémentaires. La secrétaire générale rappelle qu'un certain nombre de mesures ont déjà été mises en place depuis 2013.

Les 11 mesures principales présentées par Anne-Geneviève Bütikofer/secrétaire générale de la FMH sont les suivantes: 1) Réduction du nombre de membres du Comité central de 9 à 8; 2) Réduction du nombre annuel d'Assemblées des délégués de 6 à 4; 3) Prise en charge des indemnités de séance des délégués de l'AD par les sociétés délégantes; 4) Prise en charge à 50\% des indemnités de séance des commissions par les sociétés délégantes; 5) Augmentation des cotisations de membres; 6) Suppression du soutien annuel au centre d'information toxicologique Tox Info Suisse; 7) Potentiel d'économie de la division ICT dans les projets de base, le fonctionnement, les coûts de software et de licences, les coûts liés aux prestations des fournisseurs externes en matière de hardware et software; 8) Réduction des horaires du conseil juridique par téléphone; 9) Recettes supplémentaires pour le Bureau d'expertises extrajudiciaires en augmentant la taxe administrative de 600 à 1000 francs; 10) Externalisation des mandats de la division eHealth; 11) Maintien de la division Promotion de la santé et prévention avec des économies de CHF 37000 en réduisant les activités.

Anne-Geneviève Bütikofer/secrétaire générale de la FMH remercie tous les collaborateurs et Emanuel Waeber/ directeur administratif et financier pour leur engagement associé à une importante charge de travail supplémentaire. 
Jürg Schlup/président de la FMH remercie également tous les chefs de division et de service, et en particulier Anne-Geneviève Bütikofer et Emanuel Waeber qui n'ont pas ménagé leurs efforts.

\subsection{Procédure à suivre pour le vote des mesures de stabilisation budgétaire}

Comme aucune question n'est posée et que personne ne conteste l'objet à traiter, le président informe de deux propositions déposées en dernière minute. Il propose de discuter la proposition Nadig au point 2.2.2.2 et la proposition Leupold après le vote du bloc des mesures restantes. Tous les deux sont d'accord avec cette manière de procéder.

Pour que la Chambre médicale puisse se prononcer dans un délai raisonnable sur les mesures de stabilisation budgétaire, le Comité central propose un vote en trois étapes:

Proposition du Comité central:

Les délégués à la Chambre médicale valident le déroulement suivant pour voter les mesures de stabilisation du budget de la FMH:

1. Tout d'abord, le vote successif de chacune des 11 mesures les plus importantes.

2. Ensuite, parmi les mesures qui restent, le vote de celles pour lesquelles des propositions ont été déposées.

3. Enfin, le vote en une fois des quelque $100 \mathrm{me}$ sures restantes.

Décision:

La proposition est acceptée par 100 oui, 3 non et 1 abstention.

\subsection{Mesures de stabilisation budgétaire}

\subsubsection{Vote des 11 mesures les plus importantes}

Mesure 1: Comité central - Réduction du nombre de membres

Le Comité central propose une réduction de 9 à 8 membres tandis que le VEDAG a déposé une proposition qui prévoit de passer de 9 à 7 membres.

Hans-Anton Vogel/coprésident du VEDAG soutient l'objectif d'une FMH financièrement forte, à atteindre aussi bien par des économies que par une augmentation des cotisations de membres. Mais pour le VEDAG, les modèles de direction décidés en 2011 par la Chambre médicale sont tout aussi importants. Ceux-ci prévoient 6 départements, chacun ayant sa propre direction. Si la Chambre médicale opte pour un Comité central à 7 membres, il serait alors possible de mettre en œuvre cette ancienne décision de la ChM et de viser des économies effectives. Il demande le soutien de cette proposition.
Christoph Bosshard/CC FMH comprend la proposition du VEDAG. En sa qualité de membre du Comité central, il se demande cependant quelle est la présence que la ChM et les membres de la FMH attendent des responsables de département. Le décès d'Ernst Gähler a montré les limites de la répartition de ses attributions aux autres membres du Comité central: en nombre réduit, il a été difficile d'accomplir avec efficacité les tâches concernant les organisations de base ou la presse, et d'être présents et efficaces aux séances. Pour les membres du Comité central, il devient de plus en plus difficile de concilier leur activité médicale à la présence souhaitée. Ce qui soulève une nouvelle fois la question de la possibilité de déléguer aux chefs de division.

Peter Wiedersheim/SG revient sur les modèles de direction votés en 2011 et mentionne que le Comité central devrait majoritairement avoir une activité stratégique et laisser la gestion opérationnelle à un secrétariat général fort. Si par exemple les départements eHealth et Tarifs étaient chacun des centres de compétences, les deux doubles directions pourraient être évitées, des coûts économisés et les processus définis de manière plus efficace.

Le président passe d'abord au vote de la proposition du Comité central versus celle du VEDAG. Les délégués votent ensuite entre la proposition retenue et le nombre actuel de membres du Comité central.

Proposition du Comité central:

Le Comité central propose une réduction de 9 à 8 membres.

Proposition du VEDAG:

Réduction du nombre de membres du Comité central de 9 à 7 membres, mise en œuvre au plus tard après la ChM du 28 avril 2016.

Décision:

La proposition du VEDAG recueille 72 voix, celle du Comité central 55 voix, pour 3 abstentions.

La proposition du VEDAG est acceptée.

La proposition du VEDAG (7) versus le nombre actuel de membres du Comité central (9).

Proposition du VEDAG versus la situation actuelle: Réduction du nombre de membres du Comité central de 9 à 7 membres, mise en œuvre au plus tard après la ChM du 28 avril 2016.

Décision:

La proposition du VEDAG est acceptée par 130 oui et sans opposition.

Mesure 2: Assemblée des délégués -

Nombre de séances

Pour cette mesure, le Comité central et le VEDAG ont déposé chacun une proposition demandant de réduire 
le nombre d'Assemblées des délégués en passant de 6 à 4 séances annuelles. Comme la proposition du VEDAG est identique à celle du Comité central, ce dernier retire sa proposition en faveur de celle du VEDAG.

Urban Laffer/président fmCh rappelle que la création de l'Assemblée des délégués avec des compétences propres remonte à la réforme des structures de la FMH et qu'il avait été décidé de réunir la Chambre médicale une fois par an, et exceptionnellement de la convoquer une deuxième fois la même année. Il considère la proposition du VEDAG comme un affaiblissement de l'Assemblée des délégués et souhaite entendre l'avis de son président.

Peter Wiedersheim/président de l'Assemblée des délégués déclare que les projets d'épargne pour l'Assemblée des délégués n'ont pas été discutés avec lui. Conformément au Règlement d'exécution (RE), l'Assemblée des délégués est un organe de la FMH agissant de manière autonome et elle est, en dernier lieu, responsable envers la Chambre médicale. Les Statuts formulent les nombreuses tâches de l'Assemblée des délégués et sa fonction participe au bon fonctionnement démocratique de la FMH. Pour son président, il s'agit de prendre des décisions équilibrées au sein de la FMH. L’Assemblée des délégués est un organe servant de laboratoire d'idées (think tank) en vue de débattre des décisions politiques et de montrer la réactivité du corps médical. Le défi réside dans la communication entre les membres et leur société et les instances décisionnelles. Il invite les délégués à rejeter la proposition.

Marc Müller/SSMG et Médecins de famille Suisse soutient l'avis de son préopinant. La mission de l'Assemblée des délégués est de prendre les décisions politiques de la FMH conjointement avec le Comité central. Si la fréquence de ses séances est réduite, la FMH aura toujours un temps de retard sur le rythme des déci-

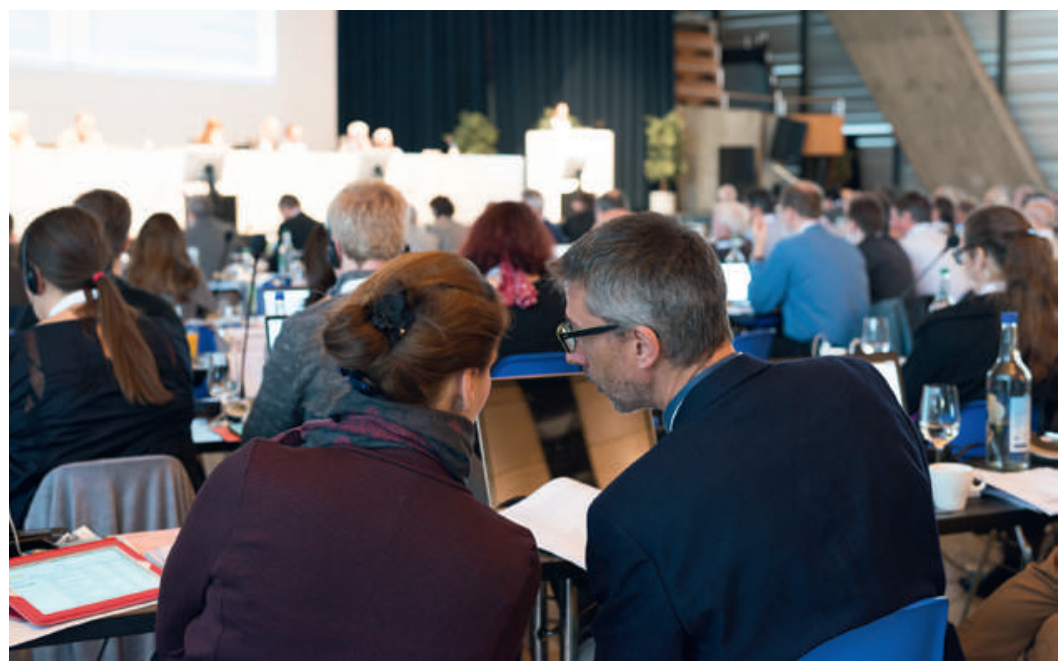

Deux délégués en pleine discussion. sions politiques. Il ne sera plus possible de défendre les intérêts des médecins au moment opportun.

Cyrill Bühlmann/ASMAC se demande si l'Assemblée des délégués, dans sa forme actuelle, a encore un sens, notamment dans le contexte des mesures de stabilisation budgétaire en cours, et si elle ne devrait pas être remplacée par un autre organe, par exemple l'ancienne conférence des présidents. Le flux d'informations serait plus simple, les organisations mieux représentées et les décisions plus largement soutenues. Sans compter que cette solution serait également moins coûteuse.

Philippe Vuillemin/VD déclare qu'il n'a toujours pas bien compris le sens de l'Assemblée des délégués par rapport à la Chambre médicale. Un dialogue devrait s'installer entre le Comité central et l'Assemblée des délégués. Mais, en prenant connaissance du procès-verbal des séances, il n’a jamais eu le sentiment jusqu'à maintenant qu'il y avait une décision incroyablement efficace de l'Assemblée des délégués qui avait changé le cours de la FMH. Comme la Chambre médicale a décidé de réduire le Comité central à 7 membres, on peut se demander si l'Assemblée des délégués ne pourrait pas reprendre une partie de ces tâches. Il soutient une réduction à 4 séances.

Hans Ulrich Iselin/AG ne souhaite pas remettre en cause l'Assemblée des délégués, mais il estime qu'elle peut aussi prouver son importance avec 4 séances.

Josef Widler/AGZ est surpris d'entendre que la Chambre médicale est prête à affaiblir un organe démocratique pour CHF 4 par membre. Ces prochaines années, la FMH devra certainement répondre à des questions au niveau politique qui demanderont la concertation $\mathrm{du}$ corps médical. Il plaide pour le maintien de 6 séances. Christine Romann/CC FMH n'est pas convaincue par l'argument des CHF 4. Cet été, lors de son analyse, le Comité central a pesé chaque centime afin de pouvoir atteindre les objectifs de la stabilisation budgétaire. Les mesures présentées concernent également des petits montants, qui additionnés entre eux constituent une somme conséquente. Pour elle, l'Assemblée des délégués reste une structure parallèle au Comité central. Lors des ateliers suivis l'année dernière avec les organisations faîtières le modérateur, un politicien expérimenté, en a conclu que la structure actuelle est une structure adaptée pour faire face aux périodes calmes et ensoleillées. Le Comité central fera de son mieux, et là aussi avec efficacité, pour accomplir son travail à 7 membres. Concernant les mesures, elle invite à suivre partout la même réflexion. Il s'agit d'économiser là où c'est possible et non selon le principe «nice to have». Elle soutient la proposition du Comité central et du VEDAG. 
Anja Zyska Cherix/ASMAC et déléguée à l'Assemblée des délégués pense que l'AD ne peut pas demander au Comité central de réduire sa structure de travail et d'économiser sans rien faire de son côté. Elle est persuadée que l'Assemblée des délégués peut accomplir un travail efficace en quatre séances par an.

Franco Denti/TI soutient la proposition de maintenir 6 séances annuelles pour l'Assemblée des délégués. Philippe Vuillemin/VD rappelle que le Comité central a présenté un programme d'économies remarquable. Il a décidé d'accepter toutes les mesures d'économies proposées. Si on veut que la FMH se porte bien financièrement, toutes ces mesures doivent être acceptées.

Florian Leupold/VEDAG précise que le VEDAG a discuté en détail de la proposition et pesé tous les arguments, de l'importance jusqu'à l'inefficacité de l'Assemblée des délégués. Il dépose une motion d'ordre pour passer maintenant au vote de cette mesure.

La motion d'ordre est acceptée à une large majorité. Avant le vote, le président rappelle que cette mesure qui concerne un changement de Statuts requiert la majorité qualifiée des deux tiers.

\section{Proposition VEDAG:}

Le VEDAG propose de réduire le nombre d'Assemblées des délégués de 6 à 4 séances annuelles en fonction des deux séances de la Chambre médicale. Décision:

La proposition est adoptée par 94 oui, 37 non et 4 abstentions.

Cyrill Bühlmann/ASMAC revient sur son intervention et dépose la proposition suivante:

Proposition Bühlmann:

Remplacer l'Assemblée des délégués par une Conférence des présidents.

Jürg Schlup/président de la FMH attire l'attention sur le fait que cette proposition implique un changement statutaire et que les propositions ayant un impact sur les Statuts doivent être déposées dans les délais définis. De ce fait, elle ne peut pas être votée aujourd'hui mais peut être soumise à la prochaine Chambre médicale.

Cyrill Bühlmann/ASMAC retire sa proposition pour cette Chambre médicale.

Mesure 3: Assemblée des délégués -

Indemnités de séance

Pour cette mesure, le VEDAG a déposé une proposition conjointement avec l'OMCT selon laquelle les indemnités de séance doivent continuer d'être versées par l'employeur de l'Assemblée des délégués, à savoir la FMH.

Florian Leupold/VEDAG précise que la proposition du Comité central ne ferait que déplacer les coûts sans répondre au principe de causalité alors que le VEDAG et l'OMCT estiment que les indemnités doivent être liées à ce principe de causalité. En effet, si on délègue une personne à des tâches importantes, on ne peut pas attendre d'elle qu'elle reçoive ses indemnités d'une organisation tierce. La rémunération participe aussi à la reconnaissance du travail accompli régulièrement par les délégués et le président de l'Assemblée des délégués. Il saisit l'occasion pour tous les remercier.

Urban Laffer/président fmCh demande si la somme de CHF 266000 inclut déjà la décision prise de ne tenir plus que 4 séances de l'Assemblée des délégués par an et si la décision de la Chambre médicale de rémunérer les délégués CHF 180 de l'heure s'applique également si les sociétés de discipline et les organisations médicales devaient elles-mêmes s'en acquitter.

Emanuel Waeber/FMH répond qu'avec 4 séances annuelles, ce montant s'élève à CHF 170000.

Anne-Geneviève Bütikofer/secrétaire générale de la FMH répond que la hauteur des indemnités relève des sociétés délégantes. Le Secrétariat général ne donne aucune recommandation.

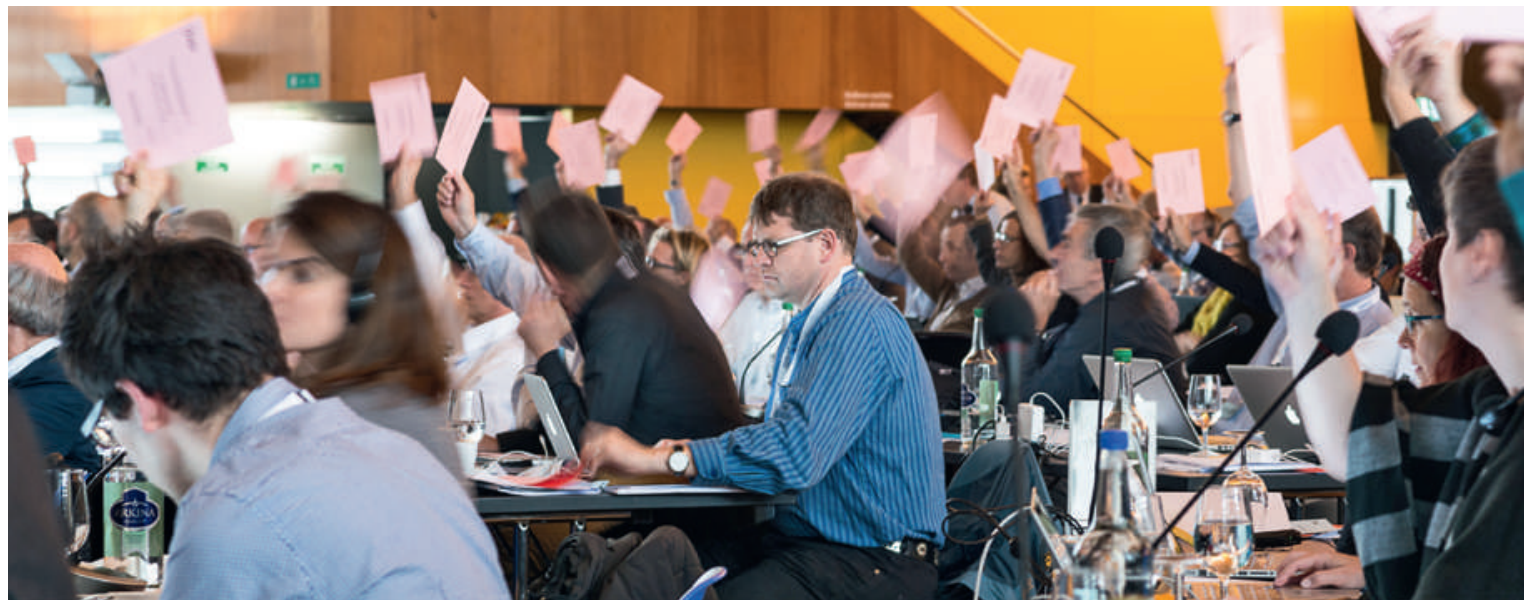

Avec leur carte de vote, les membres de la Chambre médicale indiquent s'ils acceptent ou non les différentes propositions à l'ordre du jour. 
Jürg Schlup/président de la FMH dépose une motion d'ordre pour voter cette mesure.

La motion d'ordre est acceptée à une large majorité et 2 oppositions.

Les délégués votent la proposition du Comité central versus celle du VEDAG/OMCT.

Proposition du Comité central:

Le Comité central propose que les indemnités de séance des membres de l'Assemblée des délégués soient versées par les sociétés délégantes.

Proposition du VEDAG/OMCT:

Les délégués de l'Assemblée des délégués continuent d'être indemnisés par la FMH.

Décision:

La proposition du VEDAG/OMCT recueille 101 voix, celle du Comité central 11 voix, pour 14 abstentions. La proposition du VEDAG/OMCT est acceptée. Les indemnités des délégués de l'Assemblée des délégués continueront d'être versées comme jusqu'à présent par la FMH.

Mesure 4: Commissions - Indemnités de séance

Pour cette mesure, le VEDAG propose conjointement avec l'OMCT que les indemnités de séance des commissions soient versées, comme jusqu'à présent, par la FMH. Les délégués votent la proposition du Comité central versus celle du VEDAG/OMCT.

Proposition du Comité central:

Le Comité central propose que $50 \%$ des indemnités de séance des membres des commissions soient versées par l'organisation délégante (société de discipline ou société cantonale).

Proposition du VEDAG/OMCT:

Les indemnités de séance des commissions continuent d'être versées comme jusqu'à présent par la FMH.

Décision:

La proposition du VEDAG/OMCT recueille 105 voix, celle du Comité central 16 voix, pour 2 abstentions. La proposition du VEDAG/OMCT est acceptée. Les indemnités de séance des commissions continuent d'être versées comme jusqu'à présent par la FMH.

Mesure 5: Cotisations de membre de la FMH (traitées ultérieurement)

Cette mesure sera examinée après le point «2.2.3 Vote des mesures restantes».

Mesure 6: Fondation Tox Info Suisse

Pour Angelo Cannova/AGZ, Tox Info remplit une fonction importante. Il est contre la suppression de ce soutien financier.

Hugo Kupferschmid/directeur de Tox Info rappelle que le centre est financé par les contributions de tous les partenaires du domaine de la santé. La décision du corps médical de ne pas poursuivre son soutien financier serait mal comprise et mettrait l'institution dans une situation financière difficile. Il rappelle aussi que la Chambre médicale a déjà approuvé par deux fois son soutien financier et que les conditions n'ont pas changé aujourd'hui. Le centre Tox Info fournit des prestations dont profitent non seulement les patients mais aussi le corps médical. Il demande le maintien de ce soutien financier.

Anne-Geneviève Bütikofer/secrétaire générale de la FMH signale que le centre Tox Info soutient principalement les besoins des patients (65\%) et ensuite ceux des hôpitaux dans le domaine des urgences (22\%). Seules $4 \%$ des activités sont attribuées aux médecins installés. Par conséquent, ce serait à $\mathrm{H}+$ et non à la FMH de verser cette contribution de soutien.

Prof. Jean-Michel Gaspoz/GE estime qu'on pourrait tout à fait imaginer que le centre Tox Info instaure pour le public et pour un certain nombre de prestations un numéro payant, un 0800 comme le fait Swisscom ou la Poste.

Monique Lehky Hagen/VS se prononce en faveur d'une poursuite du soutien financier. Les pertes en termes d'image pour la FMH seraient beaucoup plus importantes que les économies prévues. Elle ne mettrait pas non plus en place un numéro payant.

Thomas Heuberger/BE doute qu'il soit possible de justifier un arrêt de ce soutien et ajoute que la FMH court le risque d'un dommage collatéral irréparable.

Philippe Vuillemin/VD aimerait savoir quel est ce dommage collatéral. $4 \%$ des médecins en libre pratique utilisent ce service, c'est une réalité. Le centre Tox Info, c'est une vache sacrée, c'est un tabou du médecin suisse, il trouve donc qu'il faut un certain courage pour faire cette proposition. L'objectif, c'est de faire des économies. Il en appelle à tous d'avoir le courage de briser ce tabou. La FMH n'est pas là pour subventionner.

Proposition du Comité central:

Le Comité central propose de supprimer le soutien

financier de CHF 120000 à partir de 2017.

\section{Décision:}

La proposition est acceptée par 81 oui, 45 non et 3 abstentions.

\section{Mesure 7: ICT}

Proposition du Comité central:

Le Comité central propose un potentiel d'économie de CHF 411000 dans les projets de base, le fonctionnement, les coûts de software et de licences, les coûts liés aux prestations des fournisseurs externes en matière de hardware et software. 
Décision:

La proposition est acceptée avec 106 oui, 1 non et 1 abstention.

Mesure 8: Service juridique - Conseil juridique aux membres

Proposition du Comité central:

Le Comité central propose la réduction du conseil juridique par téléphone à une journée par semaine (conformément au modèle de l'association des propriétaires fonciers).

Décision:

La décision est acceptée par 124 oui, 1 non et 3 abstentions.

Mesure 9: Bureau d'expertises extrajudiciaires

Proposition du Comité central:

Le Comité central propose de générer des recettes supplémentaires en augmentant la taxe administrative de CHF 600 à CHF 1000. Les recettes supplémentaires s'élèvent à CHF 28000 pour environ 70 cas annuels.

Décision:

La proposition est acceptée par 124 oui, 3 non et 1 abstention.

Mesure 10: eHealth - Mandats externes

Proposition du Comité central:

Le Comité central propose l'externalisation des mandats dans le domaine eHealth.

Potentiel d'économies: CHF 21000

Décision:

La proposition est acceptée à l'unanimité.

Mesure 11: Promotion de la santé et prévention

Hans Ulrich Iselin/AG est d'avis que les activités de prévention doivent avoir un lien ciblé avec les données et les tarifs, et qu'elles doivent être intégrées dans le financement de la santé. C'est un objectif politique. La prévention mérite davantage que la politique du pot de fleur.

Christine Romann/CC FMH interprète l'intervention de son préopinant comme un soutien à son département. Elle informe que la Confédération, par exemple dans le cadre de la stratégie MNT (maladies non transmissibles), a déjà avoué vouloir inscrire la prévention dans la prise en charge médicale. Elle appelle au maintien de la division dans ce domaine.

Ricardo Torriani/AGZ estime que cette activité est sujette à discussion. La FMH est une organisation de politique professionnelle dans laquelle cette activité n'a pas sa place. Il propose de la supprimer purement et simplement de la FMH.
Christine Romann/CC FMH - après huit ans à la tête de ce département - n'accepte pas un tel point de vue. La prévention est une mission quotidienne des médecins de famille, des pédiatres et d'autres spécialistes, qui n'est cependant pas prise en compte à sa juste valeur de l'extérieur. Par ses activités, la FMH essaie de lui redonner plus de poids dans les discussions de politique de la santé. Comme les débats du groupe de travail stratégie MNT le montrent, la présence de la FMH et des autres médecins actifs dans des projets de prévention est très importante. Les avis donnés par le corps médical, dont notamment pour le financement, améliorent la place des médecins dans la prévention. Elle demande que le département soit soutenu.

Hans Jakob Furrer/Société suisse d'infectiologie souligne que la $\mathrm{FMH}$, en sa qualité d'organisation professionnelle, joue un rôle important dans la prévention précisément des maladies infectieuses. Il mentionne la thématique de la résistance aux antibiotiques, pour laquelle les médecins sont appelés à prendre position.

Proposition du Comité central:

Le Comité central propose le maintien de la division Promotion de la santé et prévention avec des économies de CHF 37000 en réduisant les activités.

Décision:

La proposition est acceptée par 130 oui, 3 non et 3 abstentions.

\subsubsection{Vote des propositions déposées sur les} mesures restantes

Après le vote des 11 mesures principales, il s'agit maintenant d'examiner les trois propositions déposées pour les mesures restantes.

\subsubsection{Proposition de l'ISFM}

Werner Bauer/président de l'ISFM mentionne qu'il avait été certifié que les discussions concernant la stabilisation budgétaire ne concerneraient pas l'ISFM. Par conséquent, ni le rapport de la taskforce, ni celui de KPMG ne contenaient de mesures allant dans ce sens. L'ISFM travaille étroitement et bien avec la FMH et lui achète chaque année des prestations dans les domaines de l'informatique, de la communication, du service juridique, etc. à hauteur d'environ CHF 2 millions. L'ISFM est tout à fait prêt à honorer ces prestations de manière adéquate et adaptée à la gestion équilibrée des deux entités. Mais deux mesures mentionnées dans le rapport du Comité central et du Secrétariat général concernent directement l'ISFM. D'une part, il s'agit d'une directive visant, en cas de surcharge, à donner la priorité aux mandats de traduction de la FMH et à externaliser les traductions de l'ISFM ce qui, en contrepartie, grèverait les comptes de l'ISFM. D'autre part, il 
s'agit du réajustement de la facturation interne qui se traduirait pour l'ISFM par des dépenses supplémentaires de CHF 135000 . Selon l'ISFM, la manière de procéder et le nouveau calcul du taux horaire ne répondent pas aux critères de l'économie d'entreprise, c'est pourquoi ces mesures sont soumises ici à la discussion.

Emanuel Waeber/FMH se réfère à une comparaison externe qui démontre que le nouveau taux proposé correspond à ce qui se fait sur le marché et est correct. Il permet en plus de garantir que les coûts internes soient couverts.

Marc Müller/Médecins de famille Suisse et Daniel Schröpfer/ASMAC estiment important que la FMH et l'ISFM puissent se mettre d'accord sur un taux horaire et qu'il serait déplorable que ces deux institutions suivent deux voies différentes, ce qui serait synonyme d'une perte importante de synergies.

Christoph Hänggeli/directeur de l'ISFM ajoute qu'il s'agit ici exclusivement de collaborateurs qui travaillent aussi bien pour la FMH que pour l'ISFM. Le principe fondamental consiste à dire que les coûts sont déterminés de telle sorte qu'aucune différence n'existe si le collaborateur est engagé par la FMH ou par l'ISFM. C'est aussi la teneur de la proposition de l'ISFM.

Le président passe au vote des propositions du Comité central versus celles de l'ISFM.

Proposition du Comité central: mesures dans le domaine de la traduction

Réduction des coûts de traduction. Priorité des textes de la FMH lors de la traduction. Les textes de l'ISFM de priorité 1 sont à donner à traduire à l'externe.

Proposition du Comité central: réajustement de la facturation interne

Taux horaires actuels:

Nouveaux taux horaires:

Collaborateur: CHF 80 Collaborateur: CHF 95 Cadre: CHF 160 Cadre: CHF 180 Expert: CHF 120

CC/SG: CHF 250 CC: CHF 200

Proposition 1 de l'ISFM: mesures dans le domaine de la traduction

Le service de traduction traite les traductions de l'ISFM selon les mêmes critères de priorité que les textes de la FMH. Aucune instruction ne peut être édictée selon laquelle les mandats de traduction de l'ISFM doivent être externalisés, c.-à-d. plus chers, pour que les mandats de traduction de la FMH soient traités plus rapidement et à meilleur marché.

Proposition 2 de l'ISFM: réajustement de la facturation interne

Les taux horaires pour la facturation interne des heures de travail entre la FMH et l'ISFM doivent être fixés de telle sorte qu'ils reflètent les coûts réels. Ces taux sont corrects si, en matière de coûts, il n'y a aucune différence que les collaborateurs qui travaillent pour les deux organisations soient engagés par la FMH ou par l'ISFM.

\section{Décision:}

Les propositions 1 et 2 de l'ISFM recueillent 96 voix, celles du Comité central 6 voix, pour 18 abstentions. Les propositions de l'ISFM sont acceptées.

\subsubsection{Proposition Jürg Nadig}

Jürg Nadig/SFSM/SSOM propose de discuter et de voter séparément les mesures d'économies qui touchent au personnel et qui ne figurent pas parmi les 11 mesures les plus importantes. La Chambre médicale doit se prononcer pour savoir s'il faut effectivement économiser sur les dépenses de personnel, le marché pour trouver du personnel très qualifié dans le domaine de la santé étant plus ou moins asséché.

Emanuel Waeber/FMH souligne que les économies touchant au personnel ont aussi été un sujet délicat pour le Comité central et les cadres. Concernant la contribution à la prévoyance professionnelle, la modification minimale proposée de passer à une répartition de 60/40 (actuellement de 65/35) est une très bonne solution. Concernant les augmentations de salaire ordinaires, toujours de l'ordre de $3 \%$ jusqu'à présent, il s'agit d'une mesure extraordinaire pour 2016. Les 3\% étaient répartis comme suit: $1 \%$ renchérissement, $1 \%$ évolution salariale dans le nouveau système de salaire et $1 \%$ pour honorer les prestations en fonction de l'entretien d'évaluation des collaborateurs. La troisième mesure concerne la réduction des coûts de formation continue de $1 \%$ à $0,5 \%$ de la masse salariale. Ce montant a cependant rarement été épuisé.

Jürg Schlup/président de la FMH explique que la proposition signifie de voter chacune des mesures concernant le personnel.

Josef Widler/AGZ dépose une motion d'ordre avant l'entrée en matière pour savoir si la proposition Nadig doit être votée car elle a été soumise au dernier moment pendant la séance.

\section{Motion d'ordre Widler}

Ceux qui veulent que la proposition Nadig soit soumise au vote l'indiquent avec leur carte de vote.

\section{Décision:}

Le vote de la proposition Nadig est rejeté par 81 non, 12 oui et 22 abstentions.

\subsubsection{Proposition Peter Wiedersheim}

Peter Wiedersheim/président de l'AD propose de maintenir la traduction simultanée pendant les Assemblées 
des délégués et de ne pas suivre la proposition du Comité central. Grâce à son introduction récente, les séances peuvent se dérouler de manière plus efficace et le "Röstigraben" ne se ressent pratiquement plus.

Daniel Schröpfer/ASMAC, Pierre Vallon/FMPP et Véronique Monnier-Cornuz/SMSR soutiennent cette proposition.

Proposition Wiedersheim:

La traduction simultanée est maintenue lors des séances de l'Assemblée des délégués.

Décision:

La proposition est acceptée par 130 oui, 2 non et 7 abstentions.

\subsubsection{Vote global de toutes les mesures restantes} Le président passe au vote des mesures restantes pour lesquelles aucune proposition n’a été déposée.

Proposition:

Vote global des mesures restantes.

Décisions:

La proposition est acceptée par 121 oui, sans opposition et 6 abstentions.

Mesure 5: Cotisations de membres de la FMH

Comme convenu, l'augmentation des cotisations de membres est débattue une fois que toutes les mesures proposées par le Comité central ont été votées.

Emanuel Waeber/FMH signale que les décisions prises aujourd'hui créent un nouveau déficit de CHF 423000 dans le budget présenté. Une augmentation de CHF 15 supplémentaires de la cotisation des catégories 1 et 2 est donc nécessaire pour que le budget reste équilibré. Il faut donc une hausse de CHF 55 (CHF 40 avaient été calculés, différence CHF 15) et non de CHF 40.

Pierre Vallon/FMPP et ancien membre de la taskforce rappelle que la participation de la FMH à EMH n'a pas encore été examinée. Cette question devrait être clarifiée avant de décider une augmentation des cotisations. Comme la proposition de Pierre Vallon n'a pas été déposée par écrit, elle n'est pas retenue.

D’après Hans-Anton Vogel/VEDAG, le VEDAG veut définir les cotisations de membres de sorte que les recettes et les dépenses de la FMH soient équilibrées. D’après les derniers calculs, cela équivaudrait à une hausse de CHF 50. Il dépose une proposition dans ce sens.

Florian Leupold/GAeSO propose de définir une cotisation adéquate dans sa motion. Par adéquate, il entend que la FMH dispose d'une marge de manœuvre financière et constitue des réserves pour intervenir en cas exceptionnel. C'était du moins la réflexion derrière la hausse de la cotisation de CHF 80.

Philippe Vuillemin/VD se prononce contre une hausse supplémentaire et soutient la proposition qui garantit un budget équilibré. Si nécessaire, les délégués pour- raient revoter une hausse des cotisations l'année prochaine.

Monique Lehky Hagen/VS propose également une augmentation de $\mathrm{CHF} 80$. Avec cette somme, la FMH pourrait constituer des réserves financières pour qu'elle puisse se faire entendre au niveau politique lors de prochains projets importants.

Daniel Schröpfer/ASMAC comprend cette volonté de réunir beaucoup d'argent, mais il propose de se mettre d'accord sur CHF 60.

Jürg Schlup/président de la FMH dépose une motion d'ordre afin de passer au vote de l'augmentation des cotisations de membres.

La motion d'ordre est acceptée à une large majorité et 1 opposition.

En raison des quatre propositions déposées, le vote se déroulera dans l'ordre suivant:

Proposition de l'ASMAC pour une augmentation des cotisations de membres de CHF 60 versus la proposition du VS/GAeSO pour une hausse de CHF 80 Décision:

La proposition de l'ASMAC recueille 80 voix, celle du VS/GAeSO 26 voix, pour 8 abstentions.

Proposition du Comité central pour une augmentation des cotisations de membres de CHF 40 versus la proposition du VEDAG pour une hausse de CHF 50 Décision:

La proposition du VEDAG recueille 108 voix, celle du Comité central 7 voix, pour 5 abstentions.

Proposition du VEDAG pour une hausse de CHF 50 versus la proposition de l'ASMAC pour une augmentation des cotisations de membres de CHF 60 Décision:

La proposition du VEDAG recueille 70 voix, celle de l'ASMAC 52 voix, pour 1 abstention.

La hausse de la cotisation de membre s'élèvera en 2016 à CHF 50.

\subsubsection{Vote final de toutes les mesures acceptées} aujourd'hui

Proposition du Comité central:

Les délégués sont d'accord avec les mesures acceptées.

\section{Décision:}

Les mesures sont acceptées par 122 oui, 1 non et 3 abstentions.

Le président remercie tous les délégués ainsi qu'AnneGeneviève Bütikofer et Emanuel Waeber pour leur excellente collaboration et leur travail constructif ayant permis d'adopter les mesures d'aujourd'hui. Le Comité central va mettre en œuvre ces mesures de manière aussi fidèle que possible. Il souhaite à tout le monde une bonne et agréable soirée. 


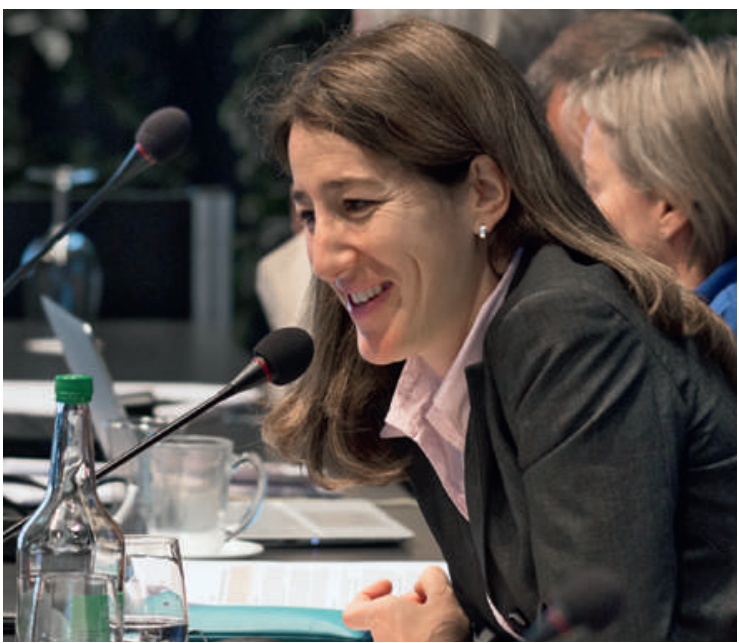

La secrétaire générale de la FMH Anne-Geneviève Bütikofer répond aux questions qui lui sont posées.

Jeudi 29 octobre 2015

\section{Accueil, communications, désignation du bureau}

Jürg Schlup/président de la FMH souhaite la bienvenue aux délégués à cette deuxième journée de la Chambre médicale. Il souhaite également la bienvenue aux invités Joachim Eder/conseiller aux Etats ZG, Hans-Rudolf Koelz/vice-président de l'ISFM, Jean Pierre Keller/vice-président de l'ISFM, Rafael Stolz/vice-président de l'ISFM, Beat Bär/directeur de FMH Services, Bruno Kesseli/rédacteur en chef du BMS, Ruedi Bienz/directeur d'EMH et Nicole Beutler/FurrerHugiPartner. D’autres invités sont également attendus: Liliane Mollet/conseillère à la protection des données de la FMH pour le point 2, Michel Meier/conseiller juridique du GAeSO pour le point 6 et Christian Peier/directeur de l'IPI pour le point 7.

Anne-Geneviève Bütikofer/secrétaire générale de la FMH donne les informations d'usage pour le déroulement de la séance et présente les différents documents qui ont été distribués aux délégués à leur arrivée et le matériel de vote pour l'élection du vice-président. Les délégués ont également reçu un dossier dans lequel ils trouveront un questionnaire du Comité central concernant la stratégie. Ils sont invités à y inscrire les défis les plus importants de la FMH pour la législature 2016 2020 et à le déposer en sortant à la fin de la séance.

Le président désigne ensuite le Bureau de la Chambre médicale, qui se compose du président, de Christine Romann/CC FMH, de la secrétaire générale, de Christoph Kreyden/responsable des élections, épaulé par la juriste Barbara Linder/ISFM, et des personnes suivantes qu'il propose d'élire comme scrutateurs: Pierre Vallon, Daniel Jud, Peter Gerritsen, Urs Schnee- berger, Duri Gianom, Daniel Ackermann, Rolf Hunkeler, Walter Kaiser, Jessika Métrailler et Daniel Schröpfer. Les scrutateurs sont élus par 150 voix et 1 abstention.

\section{Ordre du jour}

L'ordre du jour est accepté à l'unanimité.

Jürg Schlup/président de la FMH propose de clore la séance d'aujourd'hui au plus tard à 17 hoo.

\section{Décision:}

La proposition de clore la séance à 17 h00 est acceptée à l'unanimité.

Conformément à l'art. 11 al. 3 RE, aucune décision ne peut être prise et aucune élection ne peut avoir lieu après $17 \mathrm{hOO}$.

Par ailleurs, il dépose une motion d'ordre selon laquelle, conformément à l'art. 11 al. 5 RE, toutes les propositions doivent être déposées par écrit. Les délégués ont chacun le droit de faire des propositions quant aux objets de l'ordre du jour soumis à la discussion. Le texte des propositions et amendements doit être présenté au président par écrit ou exceptionnellement par oral, avant ou pendant la séance. Il souhaite supprimer cette dernière exception pour la séance d'aujourd'hui.

\section{Motion d'ordre Schlup:}

Le texte des propositions et amendements doit être présenté au président par écrit. Les propositions orales ne sont pas acceptées et ne sont pas valides.

\section{Décision:}

La motion d'ordre est acceptée par 150 oui, 1 non et 1 abstention.

\section{Allocution d'introduction du président de la FMH}

Dans son allocution, le président évoque l'aspect libéral de la profession de médecin et rappelle également les thèmes qui ont accompagné et préoccupé la FMH en 2015.

L'exercice libéral de la médecine ne se limite pas à une simple activité indépendante ni à une activité professionnelle en dehors de l'hôpital. D'ailleurs, seul un tiers environ des personnes qui exercent une profession libérale sont indépendantes. Le concept est donc beaucoup plus large et existe depuis l'Antiquité. Aujourd'hui, le Conseil fédéral définit les professions libérales comme ceci: «Toute personne qui exerce une profession libérale le fait à titre personnel et sous sa propre responsabilité. Elle ne peut être remplacée facilement, car elle fait partie intégrante de la prestation de service, qui implique surtout une relation de confiance. L'exercice d'une profession libérale suppose - toujours selon le Conseil fédéral - une qualification professionnelle élevée, une forte autonomie ainsi 
que des caractéristiques supplémentaires, comme d'avoir excellente réputation et de répondre à des exigences élevées d'ordre éthique.»

La liberté d'exercer la médecine a un corollaire: la responsabilité. Les médecins assument des responsabilités importantes, non seulement dans le cadre de leur activité médicale, mais également pour l'organisation du système de santé. Mais cette responsabilité est indissociable de leurs libertés fondamentales: la liberté de pratiquer et la liberté thérapeutique. Exercer librement la médecine, c'est prendre des décisions pour les traitements en toute indépendance, et c'est aussi garantir l'indépendance de la relation médecin-patient. Pour cela, il faut de la confiance. C'est pourquoi le corps médical lutte jour après jour contre tout ce qui pourrait entraver l'exercice libéral de la profession et contre la fragmentation des responsabilités qui diluent puis font disparaître la notion même de responsabilité. Agir de manière responsable, c'est défendre un exercice libéral et autonome de la médecine et réciproquement, préserver le caractère libéral de notre profession, c'est endosser plus de responsabilités.

L'Office fédéral de la santé publique (OFSP) veut un institut pour la qualité mais des paramètres de qualité performants font défaut. Un institut pour la qualité qui ne fait que mesurer, compter et peser, n'est pas non plus d'une grande aide. Pour améliorer encore la qualité, le corps médical souhaite des processus pertinents qu'il défend et soutient, et non une bureaucratie supplémentaire, chère et fastidieuse.

L'avenir, c'est l'avènement de la médecine individualisée et extrêmement précise dans un contexte numérique grandissant. Le corps médical doit se préparer à ce passage au numérique et à l'individualisation très précise des traitements. En matière informatique, plus aucun retour en arrière n'est possible. En médecine non plus. Une question pourrait être au cœur des débats de demain: qu'est-ce qu'un médecin doit savoir, qu'un système intelligent mis en place par les meilleurs experts ne pourra toujours pas savoir dans 30 ans? Une des réponses pour le corps médical serait de se concentrer sur la relation médecin-patient.

En Suisse, la population est très satisfaite du système de santé, comme le montrent différentes études telles que l'Euro Health Consumer Index présenté en janvier 2015 et qui place notre système de santé au deuxième rang européen en raison de sa bonne accessibilité et de la diversité de ses offres.

Suite au mandat de la Chambre médicale de 2011, les maisons d'édition Médecine et Hygiène et EMH ont réalisé en 2015 deux numéros en commun de la Revue Médicale Suisse et du Swiss Medical Weekly. Ces deux numéros ont été très réussis et le président en profite pour remercier les protagonistes Bertrand Kiefer de Médecine et Hygiène, ainsi que Nicolas Rodondi et Gérard Waeber d'EMH pour leur excellent travail. Mais en raison des coûts de production élevés et des différences culturelles, il a été décidé de renoncer à d'autres éditions communes.

La FMH continue de jouer un rôle important en politique de santé. Cette année, elle a été conviée à quatre auditions par le Parlement fédéral. La FMH reste une organisation de référence importante en matière de politique de santé et peu d'organisations ont l'occasion d'être entendues aussi souvent au cours d'une année. En ce qui concerne la structure tarifaire, l'OFSP exerce une certaine pression et provoque ainsi l'agitation du corps médical. Pour l'illustrer, la citation d'Oliver Peters, vice-directeur de l'OFSP, parue le 28 août 2015 dans la Tribune de Genève: «Nous attendons des résultats pour la fin de l'année et la division des assureurs est un mauvais signal. Nous rappelons que nous sommes prêts, le cas échéant, à intervenir, comme nous l'avons déjà fait en 2014.» Les directives de l'OFSP sont strictes et englobent une double neutralité des coûts, statique et dynamique. En guise de réponse, la FMH s'est penchée sur le cadre légal en place pour le développement du tarif. La divergence des positions l'a en effet poussée à faire le point de la situation légale sur la base d'une expertise scientifique permettant aussi d'axer et de mesurer les travaux de révision.

L'avenir de la FMH passe par la cohésion et non par la lutte entre les différentes spécialisations et cultures. L'avenir passe par des projets communs, par le renouvellement et l'innovation, par le respect mutuel. L'avenir du corps médical sera fait de devoirs, d'obligations et de transparence.

\section{Modifications des Statuts, du Règlement d'exécution et du Code de déontologie}

2.1 Modification des Statuts et du Règlement d'exécution de la FMH concernant la protection des données et la fonction de conseiller à la protection des données (Statuts: art. 12, 12a, 12b, 21, 49 et 56; Règlement d'exécution: art. $30^{\text {bis}}$ ) Jürg Schlup/président de la FMH souhaite la bienvenue à Madame Liliane Mollet/Master of Law et directrice d'insecor pour traiter de ce point à l'ordre du jour. Il rappelle les exigences accrues de la législation en matière de protection des données et les énormes progrès technologiques de ces dernières années. Une évolution qui concerne au plus haut point les grandes organisations nationales comme la FMH et dans une moindre mesure les organisations faitières ou les sociétés spéciali- 
sées ou cantonales. Dans ce contexte, le Comité central s'est fait conseiller sur ces questions complexes par le Prof. Benjamin Schindler, chaire de droit public à l'Université de St-Gall, et par Hanspeter Thür, Préposé fédéral à la protection des données et à la transparence (PFPDT), et il a décidé de mettre en œuvre trois recommandations du Prof. Schindler. En premier, un conseiller à la protection des données indépendant a été nommé pour la FMH. Liliane Mollet occupe cette fonction depuis mars 2015. Ensuite, le concept de protection des données actuel a été totalement revisité et devrait être approuvé par le Comité central début 2016. Enfin, les bases juridiques concernant la protection des données et la sécurité de l'information sont complétées dans les Statuts et le RE.

Liliane Mollet/conseillère à la protection des données de la FMH illustre à l'aide d'exemples les risques liés à la technologisation et aux nouvelles tendances. La mise en réseau accrue de l'information exige la mise en place de règles claires et uniformes en matière de protection des données. Les principaux motifs de mettre à jours les Statuts et le RE résident dans le manque de transparence dans la gestion des données des membres. La FMH a bien un concept de protection des données actuellement en cours de révision et prochainement mis en ligne sur son site internet, mais personne ne le lit ou n'en comprend la portée. La protection des données sert en premier lieu à protéger la personnalité alors que la sécurité de l'information permet de protéger les informations sensibles, à savoir les données personnelles, les informations importantes d'une entreprise et de différents systèmes. D'après le PFPDT, les traitements de données les plus importants concernant les membres d'une association (notamment la transmission de données nominatives à des tiers) doivent être définis dans les Statuts. Mais une telle réglementation permettant une mise en application coordonnée et efficace fait défaut dans les Statuts de la FMH, ainsi qu'une base juridique suffisante. Un éventuel abus ou une éventuelle perte des données personnelles détenues par la FMH pourrait avoir un impact très négatif sur son image et sa réputation. Même si ce risque ne peut jamais être entièrement écarté, la FMH devrait prendre toutes les mesures nécessaires et appropriées pour répondre aux enjeux actuels et faire preuve de responsabilité en tant qu'organisation professionnelle d'envergure nationale.

Les nouvelles dispositions des Statuts et du RE ne constituent pas une révolution en soi. Elles mentionnent les principes cardinaux de la gestion des données personnelles tout en faisant la transparence sur la pratique de la FMH. Elles définissent les grandes lignes de la fonction de conseiller à la protection des données indépendant pour la FMH. Cette dernière a désormais un interlocuteur qui l'accompagne et la conseille pour une mise en œuvre coordonnée et efficace de la protection des données mais aussi lors de projets dans lesquels la protection des données est prépondérante. La FMH envoie ainsi un signal à la hauteur de sa notoriété et de son rôle en politique, en accord avec une gestion correcte et conforme à la loi des données personnelles. Au vu du droit actuel, la FMH n'a l'obligation ni de modifier ses Statuts ni de désigner un conseiller à la protection des données. C'est ce que conclut une expertise du 17 novembre 2014 du Prof. Schindler qui souligne cependant qu'au regard de sa notoriété et de sa réputation, il serait recommandé à la FMH de modifier ses Statuts et d'établir la transparence dans la gestion des données de membres, et de désigner un conseiller à la protection des données indépendant.

Liliane Mollet/conseillère à la protection des données de la $F M H$ fait remarquer qu'il a été renoncé à une formulation détaillée des nouvelles dispositions des Statuts et du RE, dans le but de laisser délibérément une marge de manœuvre pour la pratique. L'objectif visé est d'inscrire la protection des données et la sécurité de l'information dans les Statuts et le RE de la FMH pour établir la transparence nécessaire dans la gestion des données personnelles et poser un fondement pertinent à une mise en œuvre uniforme et systématique des dispositions légales.

Le nouvel article 12 des Statuts mentionne les principes fondamentaux de la gestion des données personnelles à la FMH. Le traitement de données personnelles doit servir exclusivement à remplir les tâches inscrites dans les buts de l'association et à remplir les obligations légales. L'art. 12 mentionne explicitement la pratique de la FMH concernant le droit d'interdire la transmission de ses propres données et l'applicabilité de la loi suisse sur la protection des données. La nomination d'un conseiller à la protection des données et le renvoi au concept de protection des données sont nouveaux. L'article 12a des Statuts se réfère au traitement des données de membres et d'autres données personnelles. Il crée la transparence exigée par le PFPDT en la matière. Il mentionne explicitement le traitement des données dans le domaine de la recherche, de la planification et des statistiques, qui revêtent une importance particulière pour la FMH. La teneur de la formulation correspond à celle de la loi fédérale sur la protection des données. Il précise également que la transmission de données exige explicitement une convention séparée sur la protection des données définissant clairement les modalités de la transmission et le traitement des données personnelles.

L’article $12 b$ pose les fondements de la sécurité de 
l'information à la FMH. Dans un monde de plus en plus interconnecté, il est nécessaire d'avoir une vue d'ensemble globale lorsqu'il s'agit de gérer des informations (y c. des données personnelles) et des systèmes. Cette disposition correspond à la loi fédérale sur la protection des données. Par la prise de mesures «appropriées» pour la protection de données et de systèmes, on entend une planification diligente de mesures qui tient compte du type de l'organisation, de sa taille et de ses possibilités financières.

Les autres articles des Statuts (art. 21, 49 et 56) et du RE (art. 30 ${ }^{\text {bis }}$ ) se réfèrent à la nouvelle fonction de conseiller à la protection des données et à ses tâches. Ils répondent aux exigences de la loi fédérale sur la protection des données.

Pierre Vallon/FMPP demande si les grandes sociétés de discipline doivent procéder de la même manière en matière de protection des données.

Liliane Mollet/conseillère à la protection des données de la FMH répond que les sociétés de discipline et les organisations de base sont des personnalités juridiques autonomes et qu'elles s'organisent elles-mêmes. Pour le PFPDT, il serait bien évidemment souhaitable que la protection des données soit aussi inscrite dans leurs statuts, indépendamment du fait qu'il s'agisse d'une grande ou d'une petite association. Mais aucune pratique précise n'est encore établie en Suisse. A l'avenir, le PFPDT pourrait exiger d'inscrire les dispositions sur la protection des données dans les statuts d'une association.

Stefan Schumacher/AGZ aimerait savoir quelles seraient ses responsabilités si par exemple un hacker lui volait ses données sur son ordinateur. La FMH a-t-elle l'intention de rédiger une note répertoriant tous les standards que les utilisateurs doivent respecter?

Liliane Mollet/conseillère à la protection des données de la $F M H$ estime qu'il devrait être difficile de tenir quelqu'un pour responsable si toutes les mesures de sécurité possibles ou appropriées ont été prises (devoir de diligence), p. ex. sécurité à l'entrée du réseau, à la réception de messages, protection antivirus actualisée de l'ordinateur et du système. La création de tels standards est cependant souhaitable. La FMH est actuellement en pleine phase de planification et de mise en œuvre des mesures les plus importantes.

Jürg Schlup/président de la FMH partage l'avis suggéré par Stefan Schumacher et pense que cette prestation pourrait être proposée ultérieurement. Comme Liliane Mollet l'a déjà mentionné, la FMH vient juste de commencer la mise en place de mesures concernant la protection des données. Il soutient également la recommandation d'Hans Ulrich Iselin/AG de définir une approche du haut vers le bas dans le processus qui doit déboucher sur des solutions techniques. Le Comité central va d'abord achever la première phase et ensuite planifier d'autres mesures pour la protection des données.

Avant le vote, le président rappelle que cette décision requiert la majorité qualifiée des deux tiers.

\section{Proposition:}

Les Statuts et le Règlement d'exécution de la FMH sont à compléter avec l'ajout des nouveaux articles sur la protection des données et la fonction d'un conseiller à la protection des données indépendant.

\section{Décision:}

La décision est acceptée par 150 oui, 2 non et 1 abstention.

\section{Budgets 2016 de l'ISFM, de la FMH et de la Commission de gestion}

\subsection{Budget 2016 de l'ISFM}

Werner Bauer/président de l'ISFM saisit l'occasion pour faire quelques remarques sur le budget de l'ISFM et pour informer des projets actuels et des sujets importants. Le budget de l'ISFM présente à nouveau un déficit pour 2016. Werner Bauer souligne que les sept dernières années de vaches grasses sont maintenant suivies de sept années de vaches maigres. Le déficit résulte avant tout de la mise en œuvre de projets onéreux comme le logbook électronique, la plate-forme de formation continue et la gestion des établissements de formation postgraduée. Toutefois, ce budget déséquilibré ne signifie pas la faillite de l'ISFM mais plutôt la dissolution de quelques réserves.

Pour l'heure, l'OFSP prépare l'accréditation 2018 de tous les cursus de formation postgraduée. L'ISFM recevra à nouveau le mandat de remettre des titres de spécialiste. En comparaison avec la dernière accréditation, la prochaine occasionnera probablement moins de coûts.

En raison des modifications structurelles et des changements en cours, il est devenu plus difficile de promouvoir la formation postgraduée dans les hôpitaux. L'enseignement et la formation dans les hôpitaux doivent faire face à l'importance accordée à la performance et aux questions économiques. L'ISFM tient à renforcer le soutien accordé aux formateurs; il veut aussi les motiver à développer de nouveaux projets et de nouvelles initiatives.

Le Symposium MedEd 2015 a remporté un franc succès. Plus de 200 participants ont présenté les perspectives de la formation postgraduée et échangé leurs réflexions concernant l'avenir de la formation médicale en des temps où les structures hospitalières et de la 
santé publique changent rapidement. Lors de ce symposium, le prix Award de l'ISFM pour un engagement exceptionnel en faveur de la formation postgraduée a été remis pour la deuxième fois. Par la même occasion, l'ISFM a renouvelé son appel pour le dépôt de projets en lien avec la formation postgraduée.

Werner Bauer donne ensuite des informations sur la plate-forme "Avenir de la formation médicale" et les groupes thématiques «Coordination de la formation postgrade des médecins» et «Spécialisation croissante en médecine humaine sous l'angle de l'organisation hospitalière».

Christoph Hänggeli/directeur de l'ISFM complète les informations du président en mentionnant d'autres projets aux coûts très élevés pour l'ISFM. La dernière étape a été franchie pour le logbook électronique. L’ensemble des 82 programmes de formation postgraduée est paramétré. Dans l'intervalle, 10000 utilisateurs se sont enregistrés. Sous peu, on installera également un tableau de bord (cockpit) pour les responsables des établissements de formation postgraduée afin que les médecins-chefs puissent avoir un meilleur aperçu de leurs médecins-assistants. D'autres améliorations pratiques sont prévues pour 2016. On réalisera par exemple une interface AQC. Le prochain projet électronique concerne la plate-forme de formation continue. Le premier niveau fonctionne déjà; l'ISFM entame maintenant la réalisation du deuxième niveau qui contiendra un catalogue destiné à répertorier le plus grand nombre de sessions possible ce qui, du même coup, en allégera l'administration. Le troisième projet concerne les établissements de formation postgraduée. Ces trois projets électroniques impactent fortement le budget de l'ISFM, puisque celui-ci prévoit une perte de CHF 703000 pour 2016. Ce sont avant tout les postes «Personnel» et «Projets électroniques» qui accusent d'importants écarts par rapport aux comptes 2014. Le gain résultant de la remise de titres de spécialiste est budgétisé à la même hauteur pour l'année prochaine. L'ISFM attend à nouveau un budget équilibré dès 2018 , compte tenu de la courbe des charges et des produits. Il évalue déjà les moyens d'éviter des dépenses et de générer des recettes. Il prévoit aussi de ne promouvoir des projets plus que tous les deux ou trois ans. Pour le moment, l'augmentation de la taxe de base pour les titres de spécialiste n'est pas un sujet de discussion. Mais des ressources financières sont encore envisageables chez les établissements de formation postgraduée qui ont jusqu'ici participé dans une moindre mesure aux coûts de l'ISFM. Par exemple, on pourrait prélever une taxe de certification pour tous les établissements de formation postgraduée ou uniquement pour les nouveaux. Les seules recettes sup- plémentaires déjà fixées concernent la taxe pour le diplôme de formation continue. Dès 2016, elle devra aussi être payée par les médecins qui n'obtiennent pas leur diplôme par la plate-forme de formation continue de l'ISFM.

\subsection{Budget 2016 de la FMH (sans l'ISFM)}

Emanuel Waeber/FMH attire l'attention sur le fait que la Chambre médicale avait remis au Comité central le mandat de présenter un budget équilibré pour 2016. Les mesures décidées hier ont été intégrées dans le budget actuel. Il s'agit, en l'occurrence, des décisions suivantes avec effet direct sur le budget 2016: augmentation des cotisations de membres, réduction du nombre de membres du Comité central, réduction du nombre de séances de l'Assemblée des délégués et introduction d'un pooling de projets pour garantir une mise en œuvre prioritaire et rapide des projets planifiés. En outre, le budget adapté de la FMH pour 2016 a été élaboré en tenant compte de la planification financière à moyen et long terme 2017-2019 nouvellement mise en place. La décision de la Chambre médicale du 30 octobre 2014 d'introduire un frein aux dépenses à partir du budget 2016 a également été prise en compte. Celle-ci stipule que le montant global des charges budgétisées de la FMH (sans l'ISFM) ne doit pas dépasser les produits (cotisations de membres et recettes découlant de prestations) des derniers comptes annuels clôturés et approuvés.

Le budget de la FMH pour 2016 présente un gain de CHF 508000. Après consolidation avec la perte de l'ISFM, le déficit restant s'élève malgré tout à CHF 195000. Au niveau des produits, les écarts importants par rapport à 2015 concernent les cotisations de membres. Ils sont dus au nombre de médecins travaillant à temps partiel et bénéficiant d'une réduction de cotisation. Au niveau des charges, les écarts importants par rapport à l'année précédente concernent les restitutions de cotisations aux membres, les prestations de services, les contributions de soutien et les dépenses de personnel. La somme de CHF 2,8 millions est disponible pour la mise en œuvre de projets.

Il convient d'agir pour l'application des mesures de stabilisation budgétaire qui ont été décidées, pour le contrôle des produits résultant de prestations de services et pour l'introduction du pooling de projets. Les directives rédigées pour la nouvelle gestion des projets sont déjà appliquées et la gestion des processus sera introduite au début de 2016.

\subsection{Budget 2016 de la Commission de gestion}

Emanuel Waeber/FMH informe que les dépenses de personnel de la Commission de gestion seront moins 
élevées en 2016. Le résultat des coûts sera donc inférieur de CHF 35000.

\subsection{Contributions spéciales de la FMH: NAKO (New Index)}

Urs Stoffel/CC FMH explique les raisons de renouveler le prélèvement de la contribution spéciale NAKO. Cette contribution est extrêmement importante et elle le deviendra toujours plus. La collecte de données relatives aux médecins installés est essentielle pour assurer la qualité des données du corps médical; elle est d'autant plus importante que la révision de la structure tarifaire ambulatoire se trouve dans une phase difficile. Les évaluations au niveau cantonal, régional et national permettent au corps médical de négocier sur un pied d'égalité avec les autres acteurs de la santé publique et de défendre ainsi ses intérêts. La contribution spéciale sert à financer l'infrastructure et à garantir l'exploitation de la société NewIndex SA. Elle permet ainsi au corps médical d'accéder en permanence au système NAKO en tant que base pour l'analyse et l'évaluation de données ciblées.

Ricardo Torriani/AGZ se réfère au départ d'un collaborateur chez la concurrence et il aimerait savoir si la FMH a pensé à une clause de non-concurrence dans les contrats de travail.

Urs Stoffel/CC FMH confirme ce départ. La FMH en a tiré les conséquences et elle a déjà conclu une clause de non-concurrence avec le successeur.

Christian Bernath/SSPP aimerait savoir pourquoi ce financement continue d'avoir lieu par une contribution spéciale et ne figure pas dans le budget ordinaire. Hanspeter Kuhn/chef du service juridique de la FMH explique que les Statuts prévoient deux raisons pour prélever une contribution spéciale. La première, lorsqu'il s'agit d'un projet. En effet, les projets doivent avoir un début et une fin. La seconde, lorsque la clé de répartition n'est pas semblable à celle fixée pour la cotisation de base, donc lorsque la question du montant à payer par les médecins installés et par les médecins-assistants est réglée de façon différente.

Franziska Zogg/SSMG demande ce que la contribution spéciale finance exactement.

Urs Stoffel/CC FMH répond que cette contribution spéciale finance l'infrastructure et que, de ce fait, elle ne prend en charge que l'exploitation de NewIndex et de la NAKO. Par contre, elle ne finance pas les évaluations et la consolidation des données. Il s'agit de l'infrastructure de base sans laquelle il serait impossible d'effectuer la collecte de données dans toute la Suisse. Cette contribution spéciale finance donc les outils NAKO, non pas la consolidation des données.

\subsection{Rapport de la Commission de gestion}

Adrian Sury/président de la CdG relève que les tâches attribuées à la taskforce dans le cadre des mesures de stabilisation budgétaire ont représenté une année de travail intensif pour la Commission de gestion. Il est d'autant plus satisfait des décisions prises hier et il remercie tous les délégués de leur engagement et de leur soutien. Mais les sociétés bénéficiant d'un soutien de la part de la FMH et les éditions EMH continuent d'être un sujet de préoccupation. Le Comité central a déjà entamé des discussions avec les éditions EMH. Les premiers résultats devraient être présentés à la prochaine Chambre médicale.

Comme le budget a dû être remanié sur la base des mesures décidées hier, la Commission de gestion n'a pas pu le contrôler en détail. En tant que président de la Commission de gestion, il recommande aux délégués de l'approuver.

Les délégués se prononcent comme suit sur les différentes propositions:

Proposition du Comité central: cotisations de membres pour 2016

Approuver la proposition d'augmenter comme suit les cotisations de membres pour 2016:

$\begin{array}{ll}\text { Catégories } 1 \text { et } 2 \text { de CHF } 50 & \text { nouveau CHF } 710 \\ \text { Catégorie } 3 \text { de CHF } 35 & \text { nouveau CHF } 475 \\ \text { Catégorie } 4 \text { de CHF } 25 & \text { nouveau CHF } 355 \\ \text { Catégories } 5 \text { et } 6 \text { de CHF } 13 & \text { nouveau CHF } 178 \\ \text { Catégorie } 7 \text { de CHF } 10 & \text { nouveau CHF } 142\end{array}$

\section{Décision:}

La proposition est acceptée par 144 oui, 6 non et 1 abstention.

Proposition du Comité central: maintien des taxes d'abonnement

Approuver le maintien des taxes d'abonnement avec CHF 75.00 sans les cat. 4 et 7 (EMH: CHF 50 / SMSR: CHF 20 / OMCT: CHF 5).

Décision:

La proposition est acceptée par 148 oui, 1 non et 7 abstentions.

Proposition du Comité central: maintien de la contribution spéciale NAKO

Approuver le maintien de la contribution spéciale NAKO avec CHF 40 pour les cat. 1 et 2

Décision:

La proposition est acceptée par 118 oui, 12 non et 24 abstentions.

Josef Widler/AGZ soumet à la Chambre médicale la proposition d'inscrire une provision de CHF 40000 en faveur de Tox Info dans le budget 2016 pour l'exercice 2017. Il estime que le dommage causé à l'image par une résiliation est plus important que la contribution de soutien à discuter. 
Anne-Geneviève Bütikofer/secrétaire générale de la FMH rappelle que la décision prise hier de supprimer CHF 120000 ne deviendra effective en termes budgétaires qu'en 2017. En 2016, le Tox Info obtiendra encore le soutien convenu. Effectuer une provision à l'heure actuelle n'a pas de sens et n'est pas possible.

Philippe Vuillemin/VD s'étonne de cette proposition et invite son auteur à se plier à la décision prise hier, laquelle a été prise de façon démocratique. En outre, les mesures d'économie entraînent toujours une dégradation d'image. Il est d'avis que les suppressions décidées sont nécessaires pour équilibrer le budget et il recommande de rejeter cette proposition.

Motion d'ordre Schlup:

Les délégués veulent-ils se prononcer sur la proposition Widler?

\section{Décision:}

Les délégués refusent par 102 non, 30 oui et 16 abstentions de voter sur la proposition Widler.

Les délégués passent maintenant au vote du budget consolidé 2016 de la FMH.

Proposition du Comité central:

Accepter le budget consolidé 2016 de la FMH.

\section{Décision:}

Le budget consolidé 2016 est accepté par 149 oui et 7 abstentions.

\section{Elections, élections de confirmation et de remplacement}

\subsection{Election d'un vice-président de la FMH}

Le Comité central se compose de deux vice-présidents conformément à l'art. 47 al. 1 des Statuts. Ils sont élus par la Chambre médicale parmi les membres du Comité central, conformément à l'art. 48 al. 2 des Statuts. Les membres suivants du Comité central se présentent à l'élection pour succéder au vice-président décédé:

- Christoph Bosshard

- Remo Osterwalder

- Urs Stoffel

$\mathrm{Au} 1^{\mathrm{er}}$ tour (majorité absolue de 81 voix), obtiennent des voix:

- Christoph Bosshard 69

- Remo Osterwalder 29

- Urs Stoffel 60

- Monique Gauthey 1

- Abstention 1

$\mathrm{Au} 2^{\mathrm{e}}$ tour (majorité absolue de 82 voix), obtiennent des voix:

- Christoph Bosshard 84

- Remo Osterwalder 8

- Urs Stoffel 69

- Abstentions 2

Christoph Bosshard est élu nouveau vice-président par 84 voix.

Jürg Schlup/président de la FMH félicite Christoph Bosshard pour son élection. Il remercie Urs Stoffel et Remo Osterwalder de s'être portés candidats pour la fin de la législature.

Christoph Bosshard/CC FMH remercie les délégués de leur confiance ainsi que de leur collaboration active. La FMH se trouve actuellement face à d'importants défis. En sa qualité de vice-président de la FMH, il assumera les tâches qui lui reviennent avec un grand engagement et de manière à ce que la FMH soit soudée et s'ex-

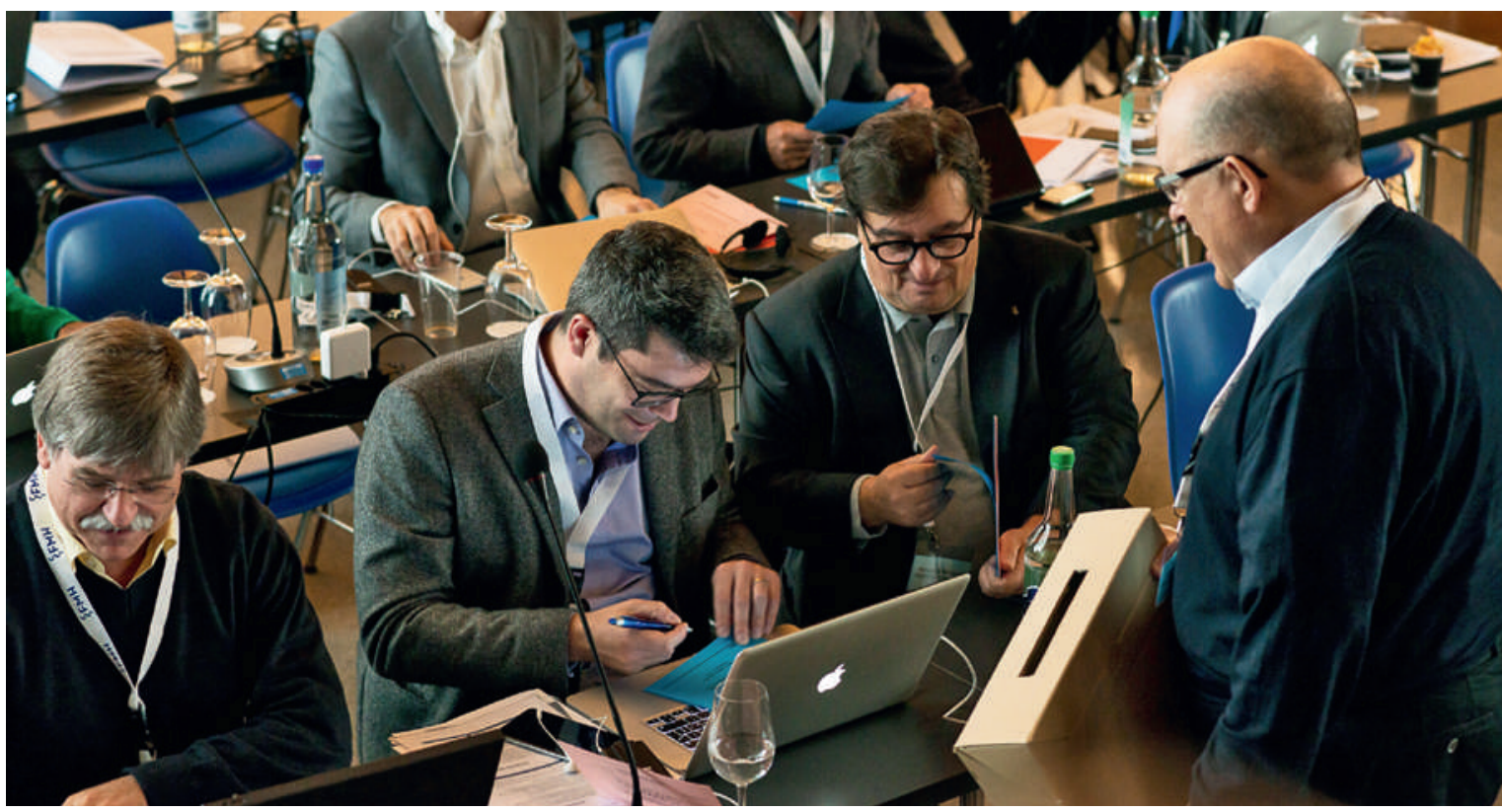

Les cartes de vote pour l'élection du vice-président de la FMH sont récoltées. 


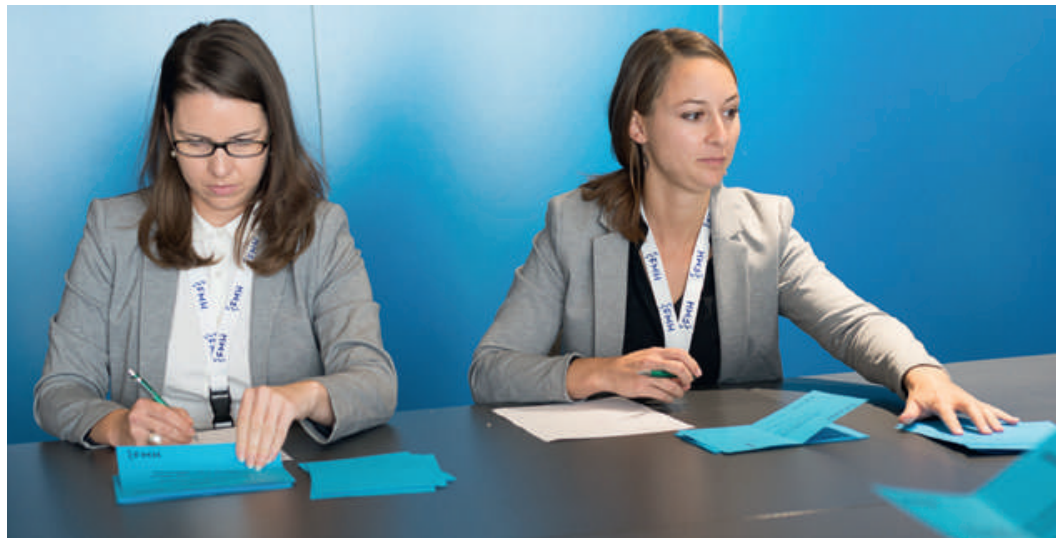

Les collaborateurs de la FMH dépouillent les bulletins avec concentration.

prime d'une seule voix vers l'extérieur. Les discussions tarifaires actuelles ne devraient pas empêcher la FMH de s'engager pour la défense des intérêts des membres et des patients.

\subsection{Election de confirmation des délégués nommés à l'Assemblée des délégués}

La Chambre médicale vote la confirmation des délégués ordinaires à l'Assemblée des délégués suivants: mws

Ancien: Marianna Bodenmann-Zanetti, Wetzikon Nouveau: Maya C. Züllig, Uster

fmch

Ancien: Ralph Schmid, Berne

Nouveau: Bernhard Egger, Zimmerwald

Ancien: Franziska Maurer-Marti, Feldbrunnen

Nouveau: Josef Emil Brandenberg, Lucerne

Proposition:

Les organisations concernées demandent à la Chambre médicale de confirmer les délégués ordinaires à l'Assemblée des délégués mentionnés ci-avant.

Décision:

Les délégués sont confirmés par 150 oui et 3 abstentions.

\subsection{Election de confirmation des délégués} remplaçants nommés à l'Assemblée des délégués

La Chambre médicale vote sur la confirmation des délégués remplaçants à l'Assemblée des délégués suivants:

SFSM

Ancien: vacant

Nouveau: Philippe A. Lyrer-Gaugler, Bâle Ann Kathrin Schwarzkopf, Berne

fmCh

Ancien: Bernhard Egger, Zimmerwald

Nouveau: Ralph Schmid, Berne

\section{SMSR}

Ancien: $\quad 2^{\mathrm{e}}$ délégué remplaçant, vacant

Nouveau: Pierre Arnold, Sion

Proposition:

Les organisations concernées demandent à la Chambre médicale de confirmer les délégués remplaçants à l'Assemblée des délégués mentionnés ci-avant.

Décision:

Les délégués remplaçants sont confirmés par 150 voix et 1 abstention.

\section{Exposé d'un intervenant invité}

Un exposé sur le thème "Décisions médicales en fin de vie» étudié dans le cadre du programme national de recherche 67 avait été prévu pour la Chambre médicale de ce jour. Comme les résultats ne sont pas encore publiés, la conférencière a annulé sa venue au dernier moment. Jürg Schlup/président de la FMH le regrette et signale qu'à sa connaissance, il est maintenant prévu de publier ces résultats en décembre 2015.

\section{Révision de la structure tarifaire ambulatoire: Tarvision et conventions}

\subsection{Etat des travaux}

En introduction, Jürg Schlup/président de la FMH cite l'art. 43 al. 4, 5 et $5^{\text {bis }}$ de la LAMal, lesquels mentionnent que les tarifs et les prix sont fixés dans des conventions conclues entre les assureurs et les fournisseurs de prestations. Si les partenaires tarifaires ne peuvent pas s'entendre, le Conseil fédéral fixe alors la structure tarifaire. Le Conseil fédéral peut en outre procéder à des adaptations de la structure tarifaire si celle-ci s'avère inappropriée et que les partenaires tarifaires ne peuvent pas s'entendre sur une révision. En d'autres termes, il faut non seulement tenir compte des positions de la FMH et des sociétés de discipline médicale mais aussi négocier avec les fournisseurs de prestations, les assureurs et les autorités.

Jürg Schlup/président de la FMH souhaite la bienvenue à Michel Meier/GAeSO, avocat, qui dirige les négociations menées par la FMH pour les nouvelles conventions tarifaires ambulatoires. Michel Meier informe de l'état des travaux du groupe de travail Conventions, dont la tâche consiste à remanier l'ensemble des conventions tarifaires. Ces conventions mettent juridiquement en pratique ce qui doit être soumis au Conseil fédéral dans le domaine de la LAMal. Par contre, le groupe de travail ne s'occupe pas de la structure et des positions tarifaires. En 2002, la FMH a conclu la convention-cadre 
avec santésuisse. En parallèle, il existe également les conventions d'adhésion cantonales que les cantons ont conclues directement avec santésuisse. La scission contractuelle des assureurs-maladie, entre santésuisse et curafutura, rend la situation plus complexe aujourd'hui qu'en 2002. Sur le plan juridique, une seule convention continue d'exister comme base et non deux. Le groupe de travail est composé actuellement de $\mathrm{H}+$, de la CTM, des assureurs qui couvrent le domaine LAA et de curafutura. En revanche, santésuisse n'est pas représentée jusqu'à présent. Le groupe de travail est en train de mettre au point un paquet contractuel contenant une convention de base comparable à la convention-cadre actuelle. Il y a de plus la structure tarifaire et la convention de normalisation. L'idée est d'envoyer au Conseil fédéral la convention de base, la structure tarifaire et la convention de normalisation pour approbation. Au niveau cantonal, la convention tarifaire restera du ressort des sociétés cantonales de médecine pour la valeur du point tarifaire. Le groupe de travail a atteint un consensus interne concernant la convention de base et en grande partie aussi la convention de normalisation. Mais les règles d'application font encore défaut dans leur ensemble. Conformément au calendrier, il devrait être possible d'ici à fin 2015 d'avoir toutes les conventions à disposition dans leurs principes afin qu'on puisse les discuter et les approuver dans les organes concernés. L'étroite collaboration avec les acteurs actuels sera maintenue.

Hans Ulrich Iselin/AG demande pourquoi la FMH parle de manière aussi complaisante de ce thème, étant donné que les assureurs-maladie boycottent la révision tarifaire. Elle devrait clairement communiquer qu'un partenaire est prêt à collaborer à ce projet complexe alors qu'un autre le boycotte systématiquement. Jürg Schlup/président de la FMH informe que $43 \%$ des assureurs participent au projet et que $57 \%$ refusent de collaborer. Les partenaires tarifaires offriront ainsi à certaines caisses la possibilité de signer la future convention.

Ricardo Torriani/AGZ relève qu'on n'entend jamais la FMH dire qu'elle rejette la neutralité des coûts. Il est d'avis que la FMH devrait se montrer plus décidée.

Urs Stoffel/CC FMH se réfère au projet «Concerto» dans lequel des experts confirmés se sont exprimés à ce sujet. La FMH y défend également sa position. La normalisation de la structure est une voie juridique envisageable montrant comment on peut satisfaire aux exigences de la neutralité des coûts. Urs Stoffel renvoie dans ce contexte à la lettre du 18 août 2014 de Pascal Strupler, directeur de l'OFSP, et à celle du 2 juin $2015 \mathrm{du}$ conseiller fédéral Alain Berset. Dans ces deux lettres, il est fait mention des conditions-cadres et aussi sou- ligné que le Conseil fédéral n’approuvera pas simplement le tarif mais qu'il procédera aussi à des corrections, si nécessaire. Le conseiller fédéral Alain Berset estime qu'il faut que la structure tarifaire envoyée pour approbation corresponde au principe de l'économicité et des coûts supportables. La FMH a répondu à ces deux lettres en prenant position. Dans sa réponse à l'interpellation du conseiller national Thomas Weibel, le Conseil fédéral note ceci au point 4: «Si, au final, l'augmentation des coûts s'avère inévitable pour l'AOS (en raison, par ex., d'un traitement de meilleure qualité ou d'une variation des coûts de revient), celle-ci doit être très limitée en raison de la viabilité financière de l'ensemble du système (principe d'équité). Dans les décisions qu'il a prises jusqu'ici, le Conseil fédéral a toujours estimé que la viabilité financière de l'ensemble du système doit en principe primer sur la justification du tarif du point de vue de l'économie d'entreprise." L'objectif est d'atteindre une structure tarifaire acceptable par tous. A cet effet, il est nécessaire de parvenir à un consensus aussi bien à la FMH que chez les partenaires tarifaires. Comme on l'a déjà mentionné, le Conseil fédéral pourra effectuer des modifications, c'est-à-dire qu'il pourra supprimer des chapitres, modifier des minutages, etc. Le calendrier prévoit la possibilité de terminer la nomenclature dans les équipes spécialisées à la mi-novembre 2015. En cas de divergences, on cherchera une solution consensuelle. Le calcul des unités fonctionnelles devrait également être établi à

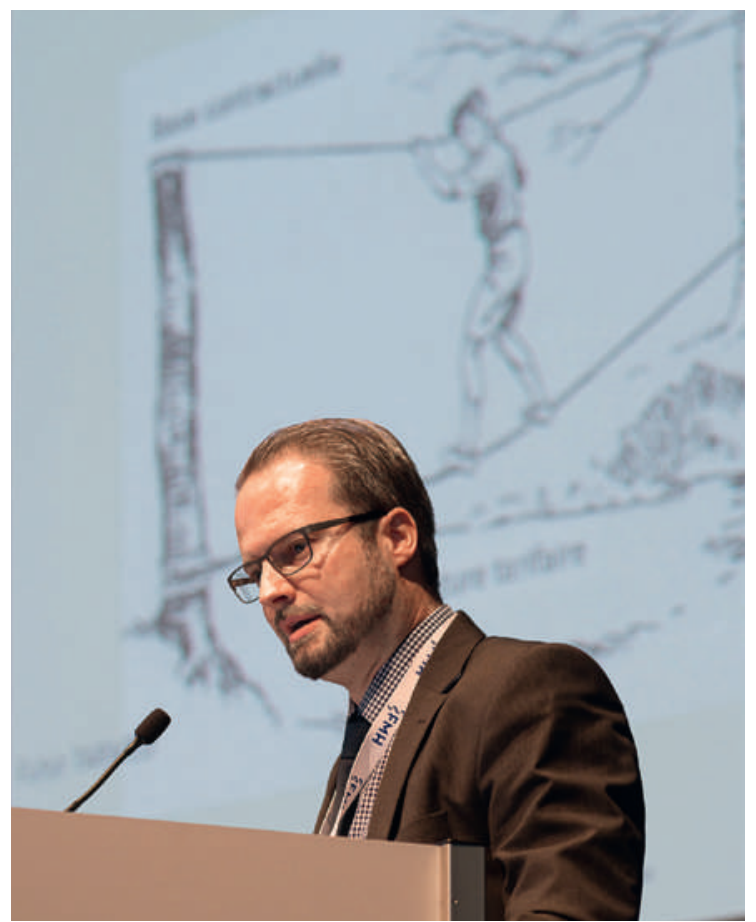

Me Michel Meier de Soleure présente les travaux du groupe de travail dédié aux conventions tarifaires. 
fin novembre 2015. Les prestations au temps et à l'acte restent le sujet le plus controversé. L’Assemblée des délégués a fixé les critères pour ces deux formes de rémunération lors de sa séance du 24 juin 2015. Les prestations hospitalières n'apparaîtront plus dans la structure tarifaire ambulatoire révisée, mais elles pourront encore être consultées dans la version 1.08.00_BR pour le calcul de la part DRG pour les médecins agréés. En outre, les prestations rares seront regroupées et simplifiées de façon judicieuse.

Il reste encore un autre défi, celui de remplacer les valeurs intrinsèques quantitatives (inscrite dans la structure tarifaire) par un facteur spécifique à chaque médecin en dehors de la structure tarifaire. Le Comité central et l'organe ad hoc «Valeurs intrinsèques» n'ont pas réussi à s'entendre sur une solution objective et adaptée, intégrée dans la structure tarifaire. Le Comité central a tenté de présenter une solution acceptable en proposant un modèle de substitution. De toute part, on souhaite un barème spécifique pour les prestations médicales avec une solution praticable à l'extérieur de la structure tarifaire. Les valeurs intrinsèques quantitatives seront fixées à 1 dans le domaine ambulatoire et remplacées par une réglementation externe à la structure tarifaire. Concernant les valeurs intrinsèques qualitatives, on était initialement parti du principe qu'on n'en avait plus besoin et qu'on pouvait totalement les supprimer. Au cours de la révision, il est cependant apparu que cette suppression n'était pas possible parce que des valeurs intrinsèques qualitatives devaient justement être attribuées à certaines prestations. Le 3 septembre 2015, l'Assemblée des délégués a adopté les critères relatifs à la gestion des valeurs intrinsèques qualitatives.

Si l'on ne parvient à aucun accord au sein des équipes spécialisées, deux propositions seront à examiner: celle des partenaires tripartites de la révision et celle des sociétés de discipline médicale. Il reviendra en dernier lieu au Comité central de trancher. Une séance spéciale du Comité central est planifiée en décembre 2015. Les travaux de traduction commenceront ensuite. La procédure de consultation démarrera en janvier 2016 et durera jusqu'à fin février 2016. L’Assemblée des délégués élaborera en mars 2016 sa recommandation à l'intention de la Chambre médicale qui se tiendra en avril 2016. L'envoi effectif de la structure tarifaire ne sera possible qu'à fin juin 2016 après une éventuelle votation générale. Ensuite, le Conseil fédéral aura six mois à disposition pour examiner la structure tarifaire. L'introduction de la nouvelle structure tarifaire reste prévue au $1^{\mathrm{er}}$ janvier 2017.

Après une discussion intéressante, Jürg Schlup/président de la FMH explique le processus d'adoption.
6.2 Processus d'adoption Révision de la structure tarifaire ambulatoire: votation générale L'objectif est de procéder à l'adoption de la révision de la structure tarifaire ambulatoire de manière transparente et démocratique. Le Comité central et l'Assemblée des délégués proposent à la Chambre médicale de respecter à cet effet l'ordre suivant.

\section{Proposition du Comité central:}

Pour approuver la révision de la structure tarifaire, le Comité central et l'Assemblée des délégués proposent à la Chambre médicale la procédure suivante qui prévoit que la révision tarifaire soit traitée par les organes dans l'ordre suivant:

- d'abord par l'Assemblée des délégués,

- ensuite lors d'une Chambre médicale, si nécessaire lors d'une Chambre médicale extraordinaire et

- enfin par la tenue d'une votation générale (tous les membres de la FMH).

\section{Décision:}

La proposition du Comité central est acceptée par 155 oui et 2 abstentions.

Jürg Schlup/président de la FMH informe que quatre nouvelles propositions lui sont parvenues depuis la pause de midi. La première proposition concerne une demande visant à revenir sur le budget.

Michaël Hagmann/SVM souhaite que les annexes au budget 2016 soient jointes au procès-verbal.

Anne-Geneviève Bütikofer/secrétaire générale de la FMH précise que le procès-verbal n'est pas envoyé aux délégués mais qu'il est à chaque fois publié dans le BMS Elle propose de déposer les annexes remaniées relatives au budget 2016 sur myFMH/DMS.

Michaël Hagmann est d'accord avec cette solution.

\section{Institut pour l'informatisation du cabinet}

\subsection{Etat des travaux 2015}

Anne-Geneviève Bütikofer/secrétaire générale de la FMH rappelle que la Chambre médicale du 30 octobre 2014 a validé la prolongation d'un an du contrat de prestations entre la FMH et l'IPI. Un avenant 2015 au contrat de 2014 entre l'IPI et la FMH n'a pas à ce jour été signé et les annexes (partie intégrante de l'avenant) font encore défaut. L'IPI est sollicité depuis l'été 2014 afin de remettre ces documents indispensables. En absence des annexes, le Comité central a reporté l'adoption de l'avenant. Le 17 juin 2015, il a demandé une révision par le comité de suivi et validé le paiement de quatre tranches trimestrielles des coûts d'infrastructure afin de ne pas pénaliser l'IPI dans son fonctionnement. Le 
23 juillet 2015, le comité de suivi a révisé l'annexe de l'avenant. Le 27 août 2015, le Comité central a approuvé l'avenant 2015 au contrat de prestations 2014 en dépit des éléments de contrôle manquants et l'a renvoyé au groupe de travail eHealth pour l'évaluation des résultats de travail et des objectifs réalisés. Le groupe de travail eHealth informera le Comité central lors de la séance de décembre 2015.

Comme mentionné, le contrat avec l'IPI n'est pas encore signé; c'est pourquoi le Comité central a décidé de déléguer le suivi au groupe de travail eHealth afin que les experts puissent examiner le contenu des résultats de travail. Le budget ne sera vraisemblablement pas épuisé et des justificatifs concernant les fonds utilisés seront nécessaires pour le paiement final. Le comité de suivi a siégé deux fois en 2015. En raison des annexes manquantes, le Comité central a dû attendre le 14 septembre 2015 avant de pouvoir transmettre à l'IPI l'avenant 2015 au contrat de prestations 2014. Le paiement des bases de développement et des projets a été effectué par la FMH, sous réserve de la signature de l'avenant et de la recommandation du groupe de travail. La secrétaire générale de la FMH et le Comité central ne peuvent donc pas présenter de rapport intermédiaire sur les activités 2015. C'est le directeur de l'IPI qui en informera ci-après.

\subsubsection{Présentation du rapport d'activité}

Christian Peier/directeur de l'IPI informe que l'IPI s'est concentré cette année sur les principaux projets, notamment sur le projet «Going Paperless». Le projet d'intégration de l'IPI dans le département eHealth a suscité des différences de vue, et il est à présent clair que cette intégration n'aura pas lieu. L'IPI a élaboré divers documents permettant au corps médical de passer du dossier du patient sur papier au dossier informatisé. Des guides, listes de contrôle et autres outils sont désormais à la disposition des médecins. En accord avec la FMH, ces documents pourront être téléchargés sur myFMH ou sur le site de l'IPI. Il est impératif, selon lui, que le corps médical poursuive dans cette voie. Sans engagement ni participation, la FMH risque de passer à côté de ce passage au numérique.

Christian Peier informe qu'il quittera ses fonctions à la direction de l'IPI fin 2015. Il remercie le comité de l'IPI, le Comité central et Anne-Geneviève Bütikofer de leur collaboration.

\subsection{Intégration de l'IPI dans le département eHealth}

Urs Stoffel/CC de la FMH soutient l'appel de Christian Peier au sujet du numérique et assure que le département eHealth s'efforcera d'obtenir l'acceptation requise.
Le 7 juillet 2015, la Chambre médicale a approuvé le concept en vue d'intégrer l'IPI dans les structures de la FMH. La mise en œuvre concrète du concept s'est toutefois avérée plus difficile que prévu en raison de différences de vue entre l'IPI et le département eHealth. L'intégration dans le département eHealth conformément à la décision de la ChM du 30 octobre 2014 ne pouvant être mise en œuvre, le département a pris l'initiative de suivre une voie susceptible d'évoluer pour accomplir le mandat de la ChM. Le groupe de travail eHealth définit les thèmes et les projets, accompagne les projets et recommande au Comité central leur validation. La mise en œuvre et la définition de la priorité des projets relèvent du département eHealth qui, grâce à cette manière de procéder, pourra dès le $1^{\mathrm{er}}$ janvier 2016 remplir le mandat de la ChM concernant «l'informatique au cabinet». Ce mandat sera réalisé avec les ressources personnelles internes et avec des mandats externes. L’Assemblée des délégués a déjà adopté ce concept le 3 septembre 2015.

Jürg Schlup/président de la FMH informe que PierreAlain Schneider/GE a soumis une proposition sur ce point.

Pour Pierre-Alain Schneider/AMG, l'IPI est un échec et il est temps, selon lui, d'en tirer les conséquences en renonçant à son intégration. Il respecte la volonté et la proposition de compromis d'Urs Stoffel, mais refuse l'idée d'un «mariage forcé». L'intégration de l'IPI s'avère impossible et il convient de l'accepter. Il propose que chaque partie recouvre sa liberté et ne poursuive plus d'objectif commun.

Franziska Zogg/SSMG ne voit pas de différence entre la proposition de Pierre-Alain Schneider et celle du Comité central.

Pierre-Alain Schneider/AMG précise qu'il souhaite une décision définitive. L'IPI était un projet intéressant, mais la tentative d'intégration a échoué. Il le regrette, mais il est temps d'en prendre acte et de tourner la page. L'IPI doit avoir la liberté de poursuivre ses activités comme il le souhaite, tout comme la FMH.

Jürg Schlup/président de la FMH souhaite mettre un terme au débat de fond et demande aux délégués s'ils désirent entrer en matière sur la proposition Schneider.

\section{Décision:}

Les délégués décident à la grande majorité d'entrer en matière sur la proposition et de poursuivre la discussion.

Hans Ulrich Iselin/AG n'est pas certain d'avoir bien saisi les explications d'Urs Stoffel. Si des projets spécifiques étaient transmis à l'IPI sous forme de mandat, il s'agirait d'ores et déjà d'un changement de paradigme. Il prie Urs Stoffel de confirmer une nouvelle fois que des projets spécifiques pourraient être mandatés à l'IPI. 
Urs Stoffel/CC FMH souligne qu'une différence existe entre la proposition Schneider et celle du Comité central. Pierre-Alain Schneider réclame que le groupe de travail eHealth soit libre d'attribuer des mandats à qui il le souhaite et de la manière qu'il le souhaite. Il est tout à fait possible - si le groupe de travail eHealth parvient à cette conclusion - d'attribuer des mandats isolés à l'IPI ou à l'organisation qui le remplacera. Avec sa proposition, Pierre-Alain Schneider souhaite une séparation claire afin qu'il n'y ait aucune obligation d'attribuer désormais tous les mandats à l'IPI. Le Comité central et le département eHealth doivent être libres d'accomplir ce mandat sans avoir à privilégier certains mandataires.

Josef Widler/AGZ souhaite s'assurer qu'il a bien compris la proposition du Comité central. La proposition précise que le mandat de la ChM ne peut pas être accompli puisque l'intégration de l'IPI n'aura pas lieu. Par ailleurs, des mandats de prestations doivent être attribués. Mais les bénéficiaires de ces mandats ne font l'objet d'aucune mention. Au vu des discussions, il propose que le Comité central retire sa proposition en faveur de la proposition Schneider.

Urs Stoffel/CC FMH préfère que les deux propositions soient soumises au vote car elles présentent une différence. Pierre-Alain Schneider souhaite tirer un trait définitif et laisser le département eHealth libre d'attribuer des mandats aux partenaires de son choix. Dès le $1^{\text {er }}$ janvier 2016, le département eHealth remplira le mandat de la ChM concernant «l'informatique au cabinet» sans être tenu de privilégier certains mandataires. Les mandats pourront être attribués à l'IPI ou à l'organisation qui le remplacera.

Daniel Schröpfer/ASMAC précise que l'ASMAC s'est toujours montrée favorable à une poursuite du développement du dossier médical informatisé et des options qui y sont liées. Au cours des deux dernières années, on a cependant réalisé que cet objectif ne pourrait pas être mené à bien avec l'IPI. Comme l'a évoqué Pierre-Alain Schneider, les mariages forcés en Suisse ne sont plus en vogue. L'ASMAC recommande donc de tirer un trait définitif et de soutenir la proposition Schneider.

Marc Müller/Médecins de famille Suisse souhaiterait maintenir la proposition du Comité central car l'IPI a précisément été fondé pour combler les lacunes du département eHealth en matière d'informatique au cabinet. Il faut donc l'assurance que le département eHealth reprendra cette question à l'avenir.

Stefan Greuter/ASMAC soutient les deux propositions car l'informatique au cabinet est un projet primordial pour la FMH. L'IPI s'est avéré un partenaire difficile. C'est pourquoi la décision de prendre une voie susceptible d'évoluer est très certainement judicieuse. Il ap- prouve également que les projets majeurs soient intégrés au groupe de travail eHealth et qu'ils soient poursuivis par la FMH. Il apportera son soutien aux deux propositions.

Les deux propositions sont désormais soumises au vote:

\section{Proposition Pierre-Alain Schneider: Abandonner l'intégration de l'IPI. \\ Décision:}

La proposition est acceptée par 130 voix et 6 abstentions.

Proposition Comité central:

Approbation par la Chambre médicale du projet de mise en œuvre présenté pour que la question de «l'informatique au cabinet» soit traitée par le département eHealth sur la base de mandats dès 2016. Décision:

La proposition est acceptée par 122 oui, 9 non et 4 abstentions.

\subsection{Réserve de la cotisation extraordinaire IPI de 2014 et 2015}

Jürg Schlup/président de la FMH informe que cette question a également fait l'objet d'une proposition. L'ASMAC demande un concept écrit pour l'utilisation des fonds en vue de la décision de la ChM en avril 2016. Anne-Geneviève Bütikofer/secrétaire générale de la FMH précise que la cotisation extraordinaire approuvée par la ChM pour 2014 et 2015 n'a pas été entièrement utilisée. Le 7 mai 2015, l'Assemblée des délégués a rejeté le prélèvement d'une contribution extraordinaire liée en vue de poursuivre les activités concernant l'informatique au cabinet dans le département eHealth. La réserve au 31 décembre 2015 s'élève à CHF 284900 et la réserve provisoire à environ CHF 384780 . La ChM est invitée ce jour à se prononcer sur l'utilisation de cette réserve d'environ CHF 669680. Trois options existent: le montant est remboursé aux membres. La deuxième option consisterait à déduire le montant de la cotisation 2016; la troisième à affecter la réserve à l'intégration, respectivement à la poursuite de l'activité informatique au cabinet par le département eHealth.

Daniel Schröpfer/ASMAC souligne qu'un concept d'utilisation des fonds fait actuellement défaut. La réserve restante n'est pas chiffrable et ne sera connue qu'après la clôture des comptes 2015. Cette réserve pourrait bien s'élever à CHF 700 000. L'ASMAC demande dans sa proposition un concept écrit pour l'utilisation des fonds qui permettra à la ChM de se prononcer à ce sujet au printemps 2016.

Jürg Schlup/président de la FMH demande aux délégués s'ils souhaitent entrer en matière sur la proposition de l'ASMAC. 
Les délégués votent l'entrée en matière par 63 voix contre 61.

Josef Widler/AGZ propose de rejeter cette proposition car elle entraîne encore plus de bureaucratie. La CdG est en charge de contrôler l'utilisation des fonds.

Pour Ricardo Torriani/AGZ, cette proposition équivaut à une motion de censure. Elle n'est actuellement nullement justifiée. La proposition doit être rejetée.

Stefan Greuter/ASMAC est, quant à lui, favorable au rejet de la proposition du Comité central. Dans le cadre du budget 2016, différentes contributions ont déjà été accordées au département eHealth pour conduire ses projets. La décision porte maintenant sur un montant de CHF 500000 ou CHF 700000 dont l'affectation n'est pas encore clarifiée.

Urs Stoffel/CC de la FMH rappelle l'approbation de l'intégration du 7 mai 2015. Cette décision concernait également le financement. Entre-temps, les projets de 2016 ont été budgétés. Un rejet de la proposition du Comité central entraverait la mise en œuvre des projets déjà planifiés.

Daniel Schröpfer/ASMAC fait remarquer que les délégués ont approuvé ce jour le budget, dont celui du département eHealth. Urs Stoffel voudrait nous faire croire qu'il ne dispose pas des moyens nécessaires. Ce n'est pas le cas. Il s'agit ici de fonds qui n'ont pas été utilisés par l'IPI. L'ASMAC souhaite que ces fonds soient affectés à des projets informatiques. C'est pourquoi elle réclame un concept correspondant. Hier, CHF 37000 ont été supprimés de la prévention, différentes sommes ont également été supprimées pour le personnel. Il s'agit ici d'un montant extrapolé de CHF 700000. La crédibilité reste primordiale.

Pour Urs Stoffel/CC FMH, la question ne porte pas ici sur un montant de CHF 700000, mais sur la réserve de la cotisation extraordinaire déjà versée. Le budget voté hier porte sur des projets comme le dossier électronique du patient, etc. Il ne prend pas en compte les projets d'informatisation du cabinet. Si ces projets doivent être réalisés dans le cadre du budget déjà voté, des réductions budgétaires sont inéluctables.

Markus Guzek/ASMAC désire savoir à qui ces projets seront attribués.

Urs Stoffel/CC FMH répond que le GT eHealth décidera de l'attribution des projets dès que le département eHealth lui aura soumis un concept correspondant. Les mandats pourront être attribués par ex. à une institution, à une haute école spécialisée, à une entreprise, etc. Hormis l'IPI, il existe d'autres institutions capables de mettre en œuvre de tels projets.

Michel Marchev/BE s'oppose à un nouveau report. Le corps médical a déjà pris aujourd'hui un certain retard en matière d'informatique. La FMH ne peut pas se per- mettre de perdre une nouvelle année. La proposition de l'ASMAC doit être rejetée.

Beat Gafner/BE soutient la proposition du Comité central. Plusieurs cantons exercent actuellement une forte pression pour introduire le dossier électronique du patient. Mais son bon fonctionnement implique qu'une grande partie des médecins en libre pratique disposent d'un dossier médical informatisé. Cela a priorité.

Hans Ulrich Iselin/AG soumet la motion d'ordre de voter immédiatement.

La motion d'ordre est approuvée à l'unanimité. La proposition du Comité central versus celle l'ASMAC sont soumises au vote.

Proposition Comité central:

Il est proposé à la ChM de valider la demande d'affectation de la réserve 2014 de CHF 284900 ainsi que la réserve non encore connue issue des comptes 2015 à l'intégration, respectivement à la poursuite de l'activité informatique au cabinet par le département eHealth.

Proposition ASMAC:

Concept écrit pour l'utilisation des fonds et en vue de la décision de la ChM en avril 2016.

\section{Décision:}

La proposition du Comité central recueille 75 voix, celle de l'ASMAC 58 voix, pour une abstention.

\section{Informations du Comité central, de l'ISFM et du Secrétariat général}

Les rapports du Comité central et du Secrétariat général ont été remis aux délégués par écrit. Ces derniers n'ont aucune question.

\section{Divers}

Jürg Schlup/président de la FMH annonce que le nouveau vice-président élu, Christoph Bosshard, entrera en fonction dès la fin de la présente ChM.

Thomas Heuberger/BE informe que la profession d'assistante médicale sera valorisée en automne par l'introduction de l'examen professionnel fédéral. Le regroupement de cabinets supprime cependant un nombre croissant de places d'apprentissage alors que de nouvelles sont nécessaires. Il en appelle donc aux personnes présentes pour contribuer à la recherche d'une solution. Patrick Scheiwiler/SG suggère de renoncer à l'allocution de l'orateur invité par manque de temps.

Pour Jürg Schlup/président de la FMH, ces exposés ne représentent pas une perte de temps. Si cette proposition répond cependant au souhait de la majorité, il sera prêt à en discuter. Il prie les délégués de lui faire part par courriel de leur opinion à ce sujet. 
Marcel Weber/Société suisse de médecine physique soumet une proposition à l'ISFM. Comme les médecinsassistants doivent aujourd'hui déjà faire face à des charges financières importantes, il conviendrait d'examiner que l'octroi d'une attestation de formation complémentaire ne fasse pas à chaque fois l'objet d'une taxe. Werner Bauer/président de l'ISFM prend acte de cette suggestion qui sera examinée.

Jürg Schlup/président de la FMH remercie l'équipe organisatrice du Secrétariat général pour l'excellente organisation et les délégués de leur coopération et participation constructive. Il souhaite à tous un bon retour.

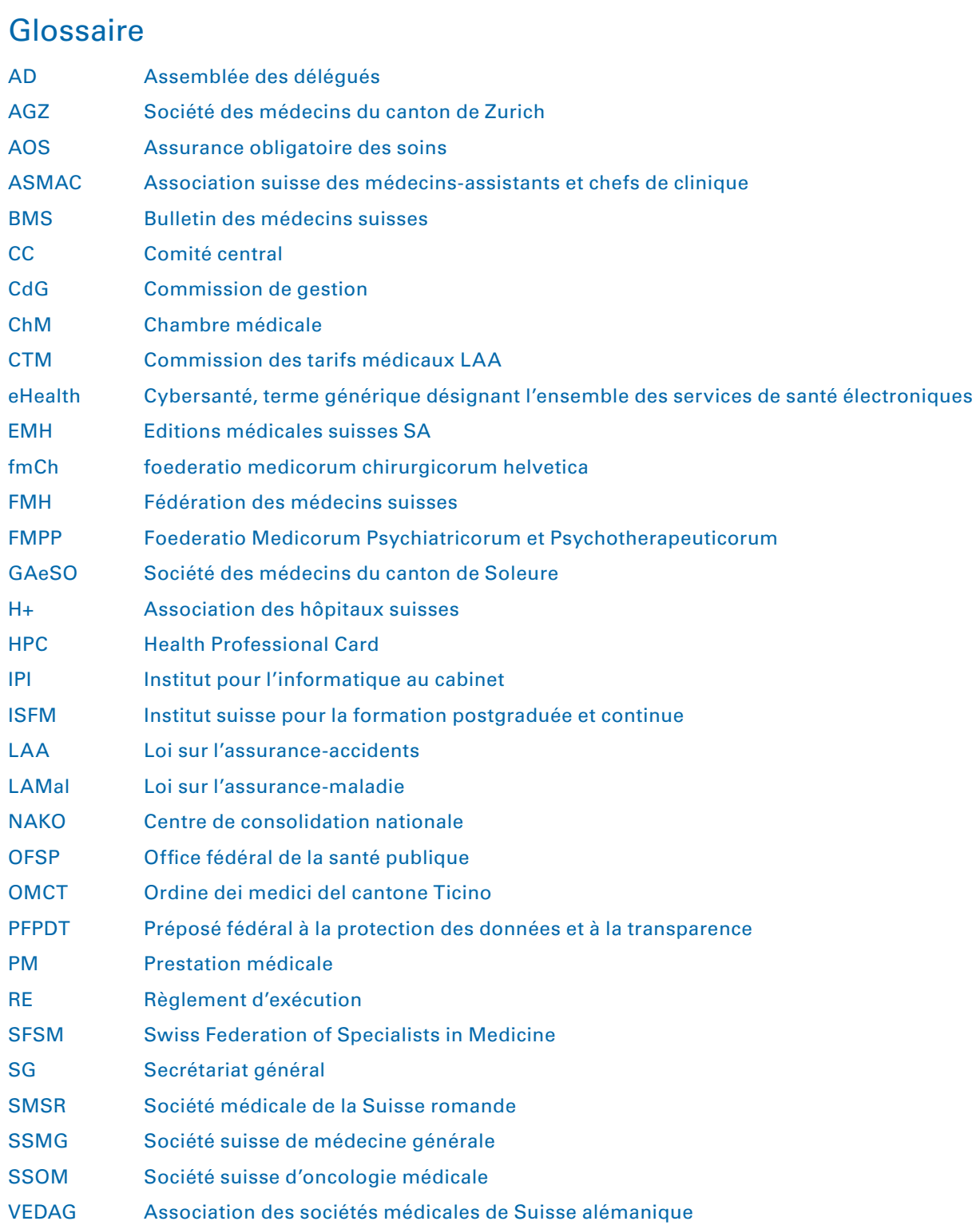

